\title{
LEFSCHETZ PENCILS, BRANCHED COVERS AND SYMPLECTIC INVARIANTS
}

\author{
DENIS AUROUX AND IVAN SMITH
}

Lectures given at the CIME summer school "Symplectic 4-manifolds and algebraic surfaces", Cetraro (Italy), September 2-10, 2003.

\begin{abstract}
Two symplectic fibrations are never exactly the same. When you have two fibrations, they might be canonically isomorphic, but when you look closely, the points of one might be numbers while the points of the other are bananas.
\end{abstract}

(P. Seidel, 9/9/03)

\section{INTRODUCTION AND BACKGROUND}

This set of lectures aims to give an overview of Donaldson's theory of linear systems on symplectic manifolds and the algebraic and geometric invariants to which they give rise. After collecting some of the relevant background, we discuss topological, algebraic and symplectic viewpoints on Lefschetz pencils and branched covers of the projective plane. The later lectures discuss invariants obtained by combining this theory with pseudo-holomorphic curve methods.

\subsection{Symplectic manifolds.}

Definition 1.1. A symplectic structure on a smooth manifold $M$ is a closed nondegenerate 2 -form $\omega$, i.e. an element $\omega \in \Omega^{2}(M)$ such that $d \omega=0$ and $\forall v \in$ $T M-\{0\}, \iota_{v} \omega \neq 0$.

For example, $\mathbb{R}^{2 n}$ carries a standard symplectic structure, given by the 2 -form $\omega_{0}=\sum d x_{i} \wedge d y_{i}$. Similarly, every orientable surface is symplectic, taking for $\omega$ any non-vanishing volume form.

Since $\omega$ induces a non-degenerate antisymmetric bilinear pairing on the tangent spaces to $M$, it is clear that every symplectic manifold is even-dimensional and orientable (if $\operatorname{dim} M=2 n$, then $\frac{1}{n !} \omega^{n}$ defines a volume form on $M$ ).

Two important features of symplectic structures that set them apart from most other geometric structures are the existence of a large number of symplectic automorphisms, and the absence of local geometric invariants.

The first point is illustrated by the following construction. Consider a smooth function $H: M \rightarrow \mathbb{R}$ (a Hamiltonian), and define $X_{H}$ to be the vector field on $M$ such that $\omega\left(X_{H}, \cdot\right)=d H$. Let $\phi_{t}: M \rightarrow M$ be the family of diifeomorphisms generated by the flow of $X_{H}$, i.e., $\phi_{0}=\mathrm{Id}$ and $\frac{d}{d t} \phi_{t}(x)=X_{H}\left(\phi_{t}(x)\right)$. Then $\phi_{t}$ is a symplectomorphism, i.e. $\phi_{t}^{*} \omega=\omega$. Indeed, we have $\phi_{0}^{*} \omega=\omega$, and

$$
\frac{d}{d t} \phi_{t}^{*} \omega=\phi_{t}^{*}\left(L_{X_{H}} \omega\right)=\phi_{t}^{*}\left(\iota_{X_{H}} \omega+\iota_{X_{H}} d \omega\right)=\phi_{t}^{*}(d(d H)+0)=0 .
$$


Therefore, the group of symplectomorphisms $\operatorname{Symp}(M, \omega)$ is infinite-dimensional, and its Lie algebra contains all Hamiltonian vector fields. An easy consequence is that $\operatorname{Symp}(M, \omega)$ acts transitively on points of $M$. This is in contrast with the case of Riemannian metrics, where isometry groups are much smaller.

The lack of local geometric invariants of symplectic structures is illustrated by two classical results of fundamental importance, which show that the study of symplectic manifolds largely reduces to topology (i.e., to discrete invariants): Darboux's theorem, and Moser's stability theorem. The first one shows that all symplectic forms are locally equivalent, in sharp contrast to the case of Riemannian metrics where curvature provides a local invariant, and the second one shows that exact deformations of symplectic structures are trivial.

Theorem 1.2 (Darboux). Every point in a symplectic manifold $\left(M^{2 n}, \omega\right)$ admits a neighborhood that is symplectomorphic to a neighborhood of the origin in $\left(\mathbb{R}^{2 n}, \omega_{0}\right)$.

Proof. We first use local coordinates to map a neighborhood of a given point in $M$ diffeomorphically onto a neighborhood $V$ of the origin in $\mathbb{R}^{2 n}$. Composing this diffeomorphism $f$ with a suitable linear transformation of $\mathbb{R}^{2 n}$, we can ensure that the symplectic form $\omega_{1}=\left(f^{-1}\right)^{*} \omega$ coincides with $\omega_{0}$ at the origin. This implies that, restricting to a smaller neighborhood if necessary, the closed 2-forms $\omega_{t}=t \omega_{1}+(1-t) \omega_{0}$ are non-degenerate over $V$ for all $t \in[0,1]$.

Using the Poincaré lemma, consider a family of 1-forms $\alpha_{t}$ on $V$ such that $\frac{d}{d t} \omega_{t}=-d \alpha_{t}$. Subtracting a constant 1 -form from $\alpha_{t}$ if necessary, we can assume that $\alpha_{t}$ vanishes at the origin for all $t$. Using the non-degeneracy of $\omega_{t}$ we can find vector fields $X_{t}$ such that $\iota_{X_{t}} \omega_{t}=\alpha_{t}$. Let $\left(\phi_{t}\right)_{t \in[0,1]}$ be the flow generated by $X_{t}$, i.e. the family of diffeomorphisms defined by $\phi_{0}=\mathrm{Id}, \frac{d}{d t} \phi_{t}(x)=X_{t}\left(\phi_{t}(x)\right)$; we may need to restrict to a smaller neighborhood $V^{\prime} \subset V$ of the origin in order to make the flow $\phi_{t}$ well-defined for all $t$. We then have

$$
\frac{d}{d t} \phi_{t}^{*} \omega_{t}=\phi_{t}^{*}\left(L_{X_{t}} \omega_{t}\right)+\phi_{t}^{*}\left(\frac{d \omega_{t}}{d t}\right)=\phi_{t}^{*}\left(d\left(\iota_{X_{t}} \omega_{t}\right)-d \alpha_{t}\right)=0
$$

and therefore $\phi_{1}^{*} \omega_{1}=\omega_{0}$. Therefore, $\phi_{1}^{-1} \circ f$ induces a symplectomorphism from a neighborhood of $x$ in $(M, \omega)$ to a neighborhood of the origin in $\left(\mathbb{R}^{2 n}, \omega_{0}\right)$.

Theorem 1.3 (Moser). Let $\left(\omega_{t}\right)_{t \in[0,1]}$ be a continuous family of symplectic forms on a compact manifold $M$. Assume that the cohomology class $\left[\omega_{t}\right] \in H^{2}(M, \mathbb{R})$ does not depend on $t$. Then $\left(M, \omega_{0}\right)$ is symplectomorphic to $\left(M, \omega_{1}\right)$.

Proof. We use the same argument as above: since $\left[\omega_{t}\right]$ is constant there exist 1-forms $\alpha_{t}$ such that $\frac{d}{d t} \omega_{t}=-d \alpha_{t}$. Define vector fields $X_{t}$ such that $\iota_{X_{t}} \omega_{t}=\alpha_{t}$ and the corresponding flow $\phi_{t}$. By the same calculation as above, we conclude that $\phi_{1}^{*} \omega_{1}=\omega_{0}$.

Definition 1.4. A submanifold $W \subset\left(M^{2 n}, \omega\right)$ is called symplectic if $\omega_{\mid W}$ is nondegenerate at every point of $W$ (it is then a symplectic form on $W$ ); isotropic if $\omega_{\mid W}=0$; and Lagrangian if it is isotropic of maximal dimension $\operatorname{dim} W=n=$ $\frac{1}{2} \operatorname{dim} M$.

An important example is the following: given any smooth manifold $N$, the cotangent bundle $T^{*} N$ admits a canonical symplectic structure that can be expressed locally as $\omega=\sum d p_{i} \wedge d q_{i}$ (where $\left(q_{i}\right)$ are local coordinates on $N$ and $\left(p_{i}\right)$ are the 
dual coordinates on the cotangent spaces). Then the zero section is a Lagrangian submanifold of $T^{*} N$.

Since the symplectic form induces a non-degenerate pairing between tangent and normal spaces to a Lagrangian submanifold, the normal bundle to a Lagrangian submanifold is always isomorphic to its cotangent bundle. The fact that this isomorphism extends beyond the infinitesimal level is a classical result of Weinstein:

Theorem 1.5 (Weinstein). For any Lagrangian submanifold $L \subset\left(M^{2 n}, \omega\right)$, there exists a neighborhood of $L$ which is symplectomorphic to a neighborhood of the zero section in the cotangent bundle $\left(T^{*} L, \sum d p_{i} \wedge d q_{i}\right)$.

There is also a neighborhood theorem for symplectic submanifolds; in that case, the local model for a neighborhood of the submanifold $W \subset M$ is a neighborhood of the zero section in the symplectic vector bundle $N W$ over $W$ (since $S p(2 n)$ retracts onto $U(n)$, the classification of symplectic vector bundles is the same as that of complex vector bundles).

\subsection{Almost-complex structures.}

Definition 1.6. An almost-complex structure on a manifold $M$ is an endomorphism $J$ of the tangent bundle $T M$ such that $J^{2}=-\mathrm{Id}$. An almost-complex structure $J$ is said to be tamed by a symplectic form $\omega$ if for every non-zero tangent vector $u$ we have $\omega(u, J u)>0$; it is compatible with $\omega$ if it is $\omega$-tame and $\omega(u, J v)=$ $-\omega(J u, v)$; equivalently, $J$ is $\omega$-compatible if and only if $g(u, v)=\omega(u, J v)$ is a Riemannian metric.

Proposition 1.7. Every symplectic manifold $(M, \omega)$ admits a compatible almostcomplex structure. Moreover, the space of $\omega$-compatible (resp. $\omega$-tame) almostcomplex structures is contractible.

This result follows from the fact that the space of compatible (or tame) complex structures on a symplectic vector space is non-empty and contractible (this can be seen by constructing explicit retractions); it is then enough to observe that a compatible (resp. tame) almost-complex structure on a symplectic manifold is simply a section of the bundle $\operatorname{End}(T M)$ that defines a compatible (resp. tame) complex structure on each tangent space.

An almost-complex structure induces a splitting of the complexified tangent and cotangent bundles: $T M \otimes \mathbb{C}=T M^{1,0} \oplus T M^{0,1}$, where $T M^{1,0}$ and $T M^{0,1}$ are respectively the $+i$ and $-i$ eigenspaces of $J$ (i.e., $T M^{1,0}=\{v-i J v, v \in T M\}$, and similarly for $T M^{0,1}$; for example, on $\mathbb{C}^{n}$ equipped with its standard complex structure, the $(1,0)$ tangent space is generated by $\partial / \partial z_{i}$ and the $(0,1)$ tangent space by $\partial / \partial \bar{z}_{i}$. Similarly, $J$ induces a complex structure on the cotangent bundle, and $T^{*} M \otimes \mathbb{C}=T^{*} M^{1,0} \oplus T^{*} M^{0,1}$ (by definition (1,0)-forms are those which pair trivially with $(0,1)$-vectors, and vice versa). This splitting of the cotangent bundle induces a splitting of differential forms into "types": $\bigwedge^{r} T^{*} M \otimes \mathbb{C}=$ $\bigoplus_{p+q=r} \bigwedge^{p} T^{*} M^{1,0} \otimes \bigwedge^{q} T^{*} M^{0,1}$. Moreover, given a function $f: M \rightarrow \mathbb{C}$ we can write $d f=\partial f+\bar{\partial} f$, where $\partial f=\frac{1}{2}(d f-i d f \circ J)$ and $\bar{\partial} f=\frac{1}{2}(d f+i d f \circ J)$ are the $(1,0)$ and $(0,1)$ parts of $d f$ respectively. Similarly, given a complex vector bundle $E$ over $M$ equipped with a connection, the covariant derivative $\nabla$ can be split into operators $\partial^{\nabla}: \Gamma(E) \rightarrow \Omega^{1,0}(E)$ and $\bar{\partial}^{\nabla}: \Gamma(E) \rightarrow \Omega^{0,1}(E)$.

Although the tangent space to a symplectic manifold $(M, \omega)$ equipped with a compatible almost-complex structure $J$ can be pointwise identified with $\left(\mathbb{C}^{n}, \omega_{0}, i\right)$, 
there is an important difference between a symplectic manifold equipped with a compatible almost-complex structure and a complex Kähler manifold: the possible lack of integrability of the almost-complex structure, namely the fact that the Lie bracket of two $(1,0)$ vector fields is not necessarily of type $(1,0)$.

Definition 1.8. The Nijenhuis tensor of an almost-complex manifold $(M, J)$ is the quantity defined by $N_{J}(X, Y)=\frac{1}{4}([X, Y]+J[X, J Y]+J[J X, Y]-[J X, J Y])$. The almost-complex structure $J$ is said to be integrable if $N_{J}=0$.

It can be checked that $N_{J}$ is a tensor (i.e., only depends on the values of the vector fields $X$ and $Y$ at a given point $)$, and that $N_{J}(X, Y)=2 \operatorname{Re}\left(\left[X^{1,0}, Y^{1,0}\right]^{(0,1)}\right)$. The non-vanishing of $N_{J}$ is therefore an obstruction to the integrability of a local frame of $(1,0)$ tangent vectors, i.e. to the existence of local holomorphic coordinates. The Nijenhuis tensor is also related to the fact that the exterior differential of a $(1,0)$ form may have a non-zero component of type $(0,2)$, so that the $\partial$ and $\bar{\partial}$ operators on differential forms do not have square zero $\left(\bar{\partial}^{2}\right.$ can be expressed in terms of $\partial$ and the Nijenhuis tensor).

Theorem 1.9 (Newlander-Nirenberg). Given an almost-complex manifold $(M, J)$, the following properties are equivalent: (i) $N_{J}=0 ;$; $\left.i i\right)\left[T^{1,0} M, T^{1,0} M\right] \subset T^{1,0} M$; (iii) $\bar{\partial}^{2}=0 ;($ iv $)(M, J)$ is a complex manifold, i.e. admits complex analytic coordinate charts.

\subsection{Pseudo-holomorphic curves and Gromov-Witten invariants.}

Pseudo-holomorphic curves, first introduced by Gromov in 1985 Gr], have since then become the most important tool in modern symplectic topology. In the same way as the study of complex curves in complex manifolds plays a central role in algebraic geometry, the study of pseudo-holomorphic curves has revolutionized our understanding of symplectic manifolds.

The equation for holomorphic maps between two almost-complex manifolds becomes overdetermined as soon as the complex dimension of the domain exceeds 1 , so we cannot expect the presence of any almost-complex submanifolds of complex dimension $\geq 2$ in a symplectic manifold equipped with a generic almost-complex structure. On the other hand, $J$-holomorphic curves, i.e. maps from a Riemann surface $(\Sigma, j)$ to the manifold $(M, J)$ such that $J \circ d f=d f \circ j$ (or in the usual notation, $\left.\bar{\partial}_{J} f=0\right)$, are governed by an elliptic PDE, and their study makes sense even in non-Kähler symplectic manifolds. The questions that we would like to answer are of the following type:

Given a compact symplectic manifold $(M, \omega)$ equipped with a generic compatible almost-complex structure $J$ and a homology class $\beta \in H_{2}(M, \mathbb{Z})$, what is the number of pseudo-holomorphic curves of given genus g, representing the homology class $\beta$ and passing through $r$ given points in $M$ (or through $r$ given submanifolds in $M$ )?

The answer to this question is given by Gromov-Witten invariants, which count such curves (in a sense that is not always obvious, as the result can e.g. be negative, and need not even be an integer). We will only consider a simple instance of the theory, in which we count holomorphic spheres which are sections of a fibration.

To start with, one must study deformations of pseudo-holomorphic curves, by linearizing the equation $\bar{\partial}_{J} f=0$ near a solution. The linearized Cauchy-Riemann operator $D_{\bar{\partial}}$, whose kernel describes infinitesimal deformations of a given curve 
$f: S^{2} \rightarrow M$, is a Fredholm operator of (real) index

$$
2 d:=\operatorname{ind} D_{\bar{\partial}}=\left(\operatorname{dim}_{\mathbb{R}} M-6\right)+2 c_{1}(T M) \cdot\left[f\left(S^{2}\right)\right] .
$$

When the considered curve is regular, i.e. when the linearized operator $D_{\bar{\partial}}$ is surjective, the deformation theory is unobstructed, and we expect the moduli space $\mathcal{M}(\beta)=\left\{f: S^{2} \rightarrow M, \bar{\partial}_{J} f=0,\left[f\left(S^{2}\right)\right]=\beta\right\}$ to be locally a smooth manifold of real dimension $2 d$.

The main result underlying the theory of pseudo-holomorphic curves is Gromov's compactness theorem (see Gr, $\mathrm{McS}, \ldots$ ):

Theorem 1.10 (Gromov). Let $f_{n}:\left(\Sigma_{n}, j_{n}\right) \rightarrow(M, \omega, J)$ be a sequence of pseudoholomorphic curves in a compact symplectic manifold, representing a fixed homology class. Then a subsequence of $\left\{f_{n}\right\}$ converges (in the "Gromov-Hausdorff topology") to a limiting map $f_{\infty}$, possibly singular.

The limiting curve $f_{\infty}$ can have a very complicated structure, and in particular its domain may be a nodal Riemann surface with more than one component, due to the phenomenon of bubbling. For example, the sequence of degree 2 holomorphic curves $f_{n}: \mathbb{C P}^{1} \rightarrow \mathbb{C P}^{2}$ defined by $f_{n}(u: v)=\left(u^{2}: u v: \frac{1}{n} v^{2}\right)$ converges to a singular curve with two degree 1 components: for $(u: v) \neq(0: 1)$, we have $\lim f_{n}(u: v)=(u: v: 0)$, so that the sequence apparently converges to a line in $\mathbb{C P}^{2}$. However the derivatives of $f_{n}$ become unbounded near $(0: 1)$, and composing $f_{n}$ with the coordinate change $\phi_{n}(u: v)=\left(\frac{1}{n} u: v\right)$ we obtain $f_{n} \circ \phi_{n}(u: v)=\left(\frac{1}{n^{2}} u^{2}: \frac{1}{n} u v: \frac{1}{n} v^{2}\right)=\left(\frac{1}{n} u^{2}: u v: v^{2}\right)$, which converges to $(0: u: v)$ everywhere except at $(1: 0)$, giving the other component (the "bubble") in the limiting curve. Therefore, it can happen that the moduli space $\mathcal{M}(\beta)$ is not compact, and needs to be compactified by adding maps with singular (nodal, possibly reducible) domains.

In the simplest case where the dimension of the moduli space is $2 d=0$, and assuming regularity, we can obtain an invariant by counting the number of points of the compactified moduli space $\overline{\mathcal{M}}(\beta)$ (up to sign). In the situations we consider, the moduli space will always be smooth and compact, but may have the wrong (excess) dimension, consisting of curves whose deformation theory is obstructed. In this case there is an obstruction bundle Obs $\rightarrow \mathcal{M}(\beta)$, whose fiber at $\left(f: S^{2} \rightarrow M\right)$ is Coker $D_{\bar{\partial}}$. In this case the invariant may be recovered as the Euler class of this bundle, viewed as an integer (the degree of Obs).

1.4. Lagrangian Floer homology. Roughly speaking, Floer homology is a refinement of intersection theory for Lagrangian submanifolds, in which we can only cancel intersection points by Whitney moves along pseudo-holomorphic Whitney discs. Formally the construction proceeds as follows.

Consider two compact orientable (relatively spin) Lagrangian submanifolds $L_{0}$ and $L_{1}$ in a symplectic manifold $(M, \omega)$ equipped with a compatible almost-complex structure $J$. Lagrangian Floer homology corresponds to the Morse theory of a functional on (a covering of) the space of arcs joining $L_{0}$ to $L_{1}$, whose critical points are constant paths.

For simplicity, we will only consider situations where it is not necessary to keep track of relative homology classes (e.g. by working over a Novikov ring), and where no bubbling can occur. For example, if we assume that $\pi_{2}(M)=\pi_{2}\left(M, L_{i}\right)=0$, then Floer homology is well-defined; to get well-defined product structures we will 
only work with exact Lagrangian submanifolds of exact symplectic manifolds (see the final section).

By definition, the Floer complex $C F^{*}\left(L_{0}, L_{1}\right)$ is the free module with one generator for each intersection point $p \in L_{0} \cap L_{1}$, and grading given by the Maslov index.

Given two points $p_{ \pm} \in L_{0} \cap L_{1}$, we can define a moduli space $\mathcal{M}\left(p_{-}, p_{+}\right)$of pseudo-holomorphic maps $f: \mathbb{R} \times[0,1] \rightarrow M$ such that $f(\cdot, 0) \in L_{0}, f(\cdot, 1) \in L_{1}$, and $\lim _{t \rightarrow \pm \infty} f(t, \tau)=p_{ \pm} \forall \tau \in[0,1]$; the expected dimension of this moduli space is the difference of Maslov indices. Assuming regularity and compactness of $\mathcal{M}\left(p_{-}, p_{+}\right)$, we can define an operator $\partial$ on $C F^{*}\left(L_{0}, L_{1}\right)$ by the formula

$$
\partial p_{-}=\sum_{p_{+}} \#\left(\mathcal{M}\left(p_{-}, p_{+}\right) / \mathbb{R}\right) p_{+},
$$

where the sum runs over all $p_{+}$for which the expected dimension of the moduli space is 1 .

In good cases we have $\partial^{2}=0$, which allows us to define the Floer homology $H F^{*}\left(L_{0}, L_{1}\right)=\operatorname{Ker} \partial / \operatorname{Im} \partial$. The assumptions made above on $\pi_{2}(M)$ and $\pi_{2}\left(M, L_{i}\right)$ eliminate the serious technical difficulties associated to bubbling (which are more serious than in the compact case, since bubbling can also occur on the boundary of the domain, a real codimension 1 phenomenon which may prevent the compactified moduli space from carrying a fundamental class, see $\mathrm{FO}^{3}$ ).

When Floer homology is well-defined, it has important consequences on the intersection properties of Lagrangian submanifolds. Indeed, for every Hamiltonian diffeomorphism $\phi$ we have $H F^{*}\left(L_{0}, L_{1}\right)=H F^{*}\left(L_{0}, \phi\left(L_{1}\right)\right)$; and if $L_{0}$ and $L_{1}$ intersect transversely, then the total rank of $H F^{*}\left(L_{0}, L_{1}\right)$ gives a lower bound on the number of intersection points of $L_{0}$ and $L_{1}$. A classical consequence, using the definition of Floer homology and the relation between $H F^{*}(L, L)$ and the usual cohomology $H^{*}(L)$, is the non-existence of compact simply connected Lagrangian submanifolds in $\mathbb{C}^{n}$.

Besides a differential, Floer complexes for Lagrangians are also equipped with a product structure, i.e. a morphism $C F^{*}\left(L_{0}, L_{1}\right) \otimes C F^{*}\left(L_{1}, L_{2}\right) \rightarrow C F^{*}\left(L_{0}, L_{2}\right)$ (well-defined in the cases that we will consider). This product structure is defined as follows: consider three points $p_{1} \in L_{0} \cap L_{1}, p_{2} \in L_{1} \cap L_{2}, p_{3} \in L_{0} \cap L_{2}$, and the moduli space $\mathcal{M}\left(p_{1}, p_{2}, p_{3}\right)$ of all pseudo-holomorphic maps $f$ from a disc with three marked points $q_{1}, q_{2}, q_{3}$ on its boundary to $M$, taking $q_{i}$ to $p_{i}$ and the three portions of boundary delimited by the marked points to $L_{0}, L_{1}, L_{2}$ respectively. We compactify this moduli space and complete it if necessary in order to obtain a well-defined fundamental cycle. The virtual dimension of this moduli space is the difference between the Maslov index of $p_{3}$ and the sum of those of $p_{1}$ and $p_{2}$. The product of $p_{1}$ and $p_{2}$ is then defined as

$$
p_{1} \cdot p_{2}=\sum_{p_{3}} \# \mathcal{M}\left(p_{1}, p_{2}, p_{3}\right) p_{3},
$$

where the sum runs over all $p_{3}$ for which the expected dimension of the moduli space is zero.

While the product structure on $C F^{*}$ defined by (11) satisfies the Leibniz rule with respect to the differential $\partial$ (and hence descends to a product structure on Floer homology), it differs from usual products by the fact that it is only associative up to homotopy. In fact, Floer complexes come equipped with a full set of higher-order 
products

$$
\mu^{n}: C F^{*}\left(L_{0}, L_{1}\right) \otimes \cdots \otimes C F^{*}\left(L_{n-1}, L_{n}\right) \rightarrow C F^{*}\left(L_{0}, L_{n}\right) \text { for all } n \geq 1,
$$

with each $\mu^{n}$ shifting degree by $2-n$. The first two maps $\mu^{1}$ and $\mu^{2}$ are respectively the Floer differential $\partial$ and the product described above. The definition of $\mu^{n}$ is similar to those of $\partial$ and of the product structure: given generators $p_{i} \in C F^{*}\left(L_{i-1}, L_{i}\right)$ for $1 \leq i \leq n$ and $p_{n+1} \in C F^{*}\left(L_{0}, L_{n}\right)$ such that $\operatorname{deg} p_{n+1}=\sum_{i=1}^{n} \operatorname{deg} p_{i}+2-n$, the coefficient of $p_{n+1}$ in $\mu^{n}\left(p_{1}, \ldots, p_{n}\right)$ is obtained by counting (in a suitable sense) pseudo-holomorphic maps $f$ from a disc with $n+1$ marked points $q_{1}, \ldots, q_{n+1}$ on its boundary to $M$, such that $f\left(q_{i}\right)=p_{i}$ and the portions of boundary delimited by the marked points are mapped to $L_{0}, \ldots, L_{n}$ respectively.

The maps $\left(\mu^{n}\right)_{n \geq 1}$ define an $A_{\infty}$-structure on Floer complexes, i.e. they satisfy an infinite sequence of algebraic relations:

$$
\left\{\begin{array}{l}
\mu^{1}\left(\mu^{1}(a)\right)=0, \\
\mu^{1}\left(\mu^{2}(a, b)\right)=\mu^{2}\left(\mu^{1}(a), b\right)+(-1)^{\operatorname{deg} a} \mu^{2}\left(a, \mu^{1}(b)\right), \\
\mu^{1}\left(\mu^{3}(a, b, c)\right)=\mu^{2}\left(\mu^{2}(a, b), c\right)-\mu^{2}\left(a, \mu^{2}(b, c)\right) \\
\quad \pm \mu^{3}\left(\mu^{1}(a), b, c\right) \pm \mu^{3}\left(a, \mu^{1}(b), c\right) \pm \mu^{3}\left(a, b, \mu^{1}(c)\right), \\
\ldots \quad
\end{array}\right.
$$

This leads to the concept of "Fukaya category" of a symplectic manifold. Conjecturally, for every symplectic manifold $(M, \omega)$ one should be able to define an $A_{\infty}$-category $\mathcal{F}(M)$ whose objects are Lagrangian submanifolds (compact, orientable, relatively spin, "twisted" by a flat unitary vector bundle); the space of morphisms between two objects $L_{0}$ and $L_{1}$ is the Floer complex $C F^{*}\left(L_{0}, L_{1}\right)$ equipped with its differential $\partial=\mu^{1}$, with (non-associative) composition given by the product $\mu^{2}$, and higher order compositions $\mu^{n}$.

The importance of Fukaya categories in modern symplectic topology is largely due to the homological mirror symmetry conjecture, formulated by Kontsevich. Very roughly, this conjecture states that the phenomenon of mirror symmetry, i.e. a conjectural correspondence between symplectic manifolds and complex manifolds ("mirror pairs") arising from a duality among string theories, should be visible at the level of Fukaya categories of symplectic manifolds and categories of coherent sheaves on complex manifolds: given a mirror pair consisting of a symplectic manifold $M$ and a complex manifold $X$, the derived categories $D \mathcal{F}(M)$ and $D^{b} \operatorname{Coh}(X)$ should be equivalent (in a more precise form of the conjecture, one should actually consider families of manifolds and deformations of categories). However, due to the very incomplete nature of our understanding of Fukaya categories in comparison to the much better understood derived categories of coherent sheaves, this conjecture has so far only been verified on very specific examples.

1.5. The topology of symplectic 4-manifolds. To end our introduction, we mention some of the known results and open questions in the theory of compact symplectic 4-manifolds, which motivate the directions taken in the later lectures.

Recall that, in the case of open manifolds, Gromov's $h$-principle implies that the existence of an almost-complex structure is sufficient. In contrast, the case of compact manifolds is much less understood, except in dimension 4 . Whereas the existence of a class $\alpha \in H^{2}(M, \mathbb{R})$ such that $\alpha^{\cup n} \neq 0$ and of an almost-complex structure already provide elementary obstructions to the existence of a symplectic 
structure on a given compact manifold, in the case of 4-manifolds a much stronger obstruction arises from Seiberg-Witten invariants. We will not define these, but mention some of their key topological consequences for symplectic 4-manifolds, which follow from the work of Taubes (Ta1, [Ta2], ... ).

Theorem 1.11 (Taubes). (i) Let $\left(M^{4}, \omega\right)$ be a compact symplectic 4-manifold with $b_{2}^{+} \geq 2$. Then the homology class $c_{1}\left(K_{M}\right)$ admits a (possibly disconnected) smooth pseudo-holomorphic representative (in particular $c_{1}\left(K_{M}\right) \cdot[\omega] \geq 0$ ). Hence, if $M$ is minimal i.e. contains no $(-1)$-spheres, then $c_{1}\left(K_{M}\right)^{2}=2 \chi(M)+3 \sigma(M) \geq 0$.

(ii) If $\left(M^{4}, \omega\right)$ splits as a connected sum $M_{1} \# M_{2}$, then one of the $M_{i}$ has negative definite intersection form.

When $b_{2}^{+}(M)=1$, Seiberg-Witten theory still has some implications. Using Gromov's characterization of the Fubini-Study symplectic structure of $\mathbb{C P}^{2}$ in terms of the existence of pseudo-holomorphic lines, Taubes has shown that the symplectic structure of $\mathbb{C P}^{2}$ is unique up to scaling. This result has been extended by Lalonde and McDuff to the case of rational ruled surfaces, where $\omega$ is determined by its cohomology class.

Remark. For any smooth connected symplectic curve $\Sigma$ in a symplectic fourmanifold $(M, \omega)$, the genus $g(\Sigma)$ is related to the homology class by the classical adjunction formula

$$
2-2 g(\Sigma)+[\Sigma] \cdot[\Sigma]=-c_{1}\left(K_{M}\right) \cdot[\Sigma],
$$

a direct consequence of the splitting $T M_{\mid \Sigma}=T \Sigma \oplus N \Sigma$. For example, every connected component of the pseudo-holomorphic representative of $c_{1}\left(K_{M}\right)$ constructed by Taubes satisfies $g(\Sigma)=1+[\Sigma] \cdot[\Sigma]$ (this is how one derives the inequality $c_{1}\left(K_{M}\right)^{2} \geq 0$ under the minimality assumption). In fact, Seiberg-Witten theory also implies that symplectic curves have minimal genus among all smoothly embedded surfaces in their homology class.

In parallel to the above constraints on symplectic 4-manifolds, surgery techniques have led to many interesting new examples of compact symplectic manifolds.

One of the most efficient techniques in this respect is the symplectic sum construction, investigated by Gompf Go1: if two symplectic manifolds $\left(M_{1}^{2 n}, \omega_{1}\right)$ and $\left(M_{2}^{2 n}, \omega_{2}\right)$ contain compact symplectic hypersurfaces $W_{1}^{2 n-2}, W_{2}^{2 n-2}$ that are mutually symplectomorphic and whose normal bundles have opposite Chern classes, then we can cut $M_{1}$ and $M_{2}$ open along the submanifolds $W_{1}$ and $W_{2}$, and glue them to each other along their common boundary, performing a fiberwise connected sum in the normal bundles to $W_{1}$ and $W_{2}$, to obtain a new symplectic manifold $M=M_{1 W_{1}} \#_{W_{2}} M_{2}$. This construction has in particular allowed Gompf to show that every finitely presented group can be realized as the fundamental group of a compact symplectic 4-manifold. This is in sharp contrast to the Kähler case, where Hodge theory shows that the first Betti number is always even.

A large number of questions remain open, even concerning the Chern numbers realized by symplectic 4-manifolds. For instance it is unknown to this date whether the Bogomolov-Miyaoka-Yau inequality $c_{1}^{2} \leq 3 c_{2}$, satisfied by all complex surfaces of general type, also holds in the symplectic case. Moreover, very little is known about the symplectic topology of complex surfaces of general type. 


\section{Symplectic Lefschetz FiBRAtions}

This section will provide a theoretical classification of symplectic 4-manifolds in algebraic terms, but we begin very humbly.

2.1. Fibrations and monodromy. Here is an easy way to build symplectic 4manifolds:

Proposition 2.1 (Thurston). If $\Sigma_{g} \rightarrow X \rightarrow \Sigma_{h}$ is a surface bundle with fiber non-torsion in homology, then $X$ is symplectic.

Proof. Let $\eta \in \Omega^{2}(M)$ be a closed 2-form representing a cohomology class which pairs non-trivially with the fiber. Cover the base $\Sigma_{h}$ by balls $U_{i}$ over which the fibration is trivial: we have a diffeomorphism $\phi_{i}: f^{-1}\left(U_{i}\right) \rightarrow U_{i} \times \Sigma_{g}$, which determines a projection $p_{i}: f^{-1}\left(U_{i}\right) \rightarrow \Sigma_{g}$.

Let $\sigma$ be a symplectic form on the fiber $\Sigma_{g}$, in the same cohomology class as the restriction of $\eta$. After restriction to $f^{-1}\left(U_{i}\right) \simeq U_{i} \times \Sigma_{g}$, we can write $p_{i}^{*} \sigma=\eta+d \alpha_{i}$ for some 1-form $\alpha_{i}$ over $f^{-1}\left(U_{i}\right)$. Let $\left\{\rho_{i}\right\}$ be a partition of unity subordinate to the cover $\left\{U_{i}\right\}$ of $\Sigma_{h}$, and let $\tilde{\eta}=\eta+\sum_{i} d\left(\left(\rho_{i} \circ f\right) \alpha_{i}\right)$. The 2-form $\tilde{\eta}$ is welldefined since the support of $\rho_{i} \circ f$ is contained in $f^{-1}\left(U_{i}\right)$, and it is obviously closed. Moreover, over $f^{-1}(p)$, we have $\tilde{\eta}_{\mid f^{-1}(p)}=\eta_{\mid f^{-1}(p)}+\sum_{i} \rho_{i}(p) d \alpha_{i \mid f^{-1}(p)}=$ $\sum_{i} \rho_{i}(p)\left(\eta+d \alpha_{i}\right)_{\mid f^{-1}(p)}=\sum_{i} \rho_{i}(p)\left(p_{i}^{*} \sigma\right)_{\mid f^{-1}(p)}$. Since a positive linear combination of symplectic forms over a Riemann surface is still symplectic, the form $\tilde{\eta}$ is nondegenerate on every fiber.

At any point $x \in X$, the tangent space $T_{x} X$ splits into a vertical subspace $V_{x}=$ Ker $d f_{x}$ and a horizontal subspace $H_{x}=\left\{v \in T_{x} X, \tilde{\eta}\left(v, v^{\prime}\right)=0 \forall v^{\prime} \in V_{x}\right\}$. Since the restriction of $\tilde{\eta}$ to the vertical subspace is non-degenerate, we have $T_{x} X=H_{x} \oplus V_{x}$. Letting $\kappa$ be a symplectic form on the base $\Sigma_{h}$, the 2 -form $f^{*} \kappa$ is non-degenerate over $H_{x}$, and therefore for sufficiently large $C>0$ the 2 -form $\tilde{\eta}+C f^{*} \kappa$ defines a global symplectic form on $X$.

The cohomology class of the symplectic form depends on $C$, but the structure is canonical up to deformation equivalence. The hypothesis on the fiber is satisfied whenever $g \neq 1$, since $c_{1}\left(T X^{\text {vert }}\right)$ evaluates non-trivially on the fiber. That some assumption is needed for $g=1$ is shown by the example of the Hopf fibration $T^{2} \rightarrow S^{1} \times S^{3} \rightarrow S^{2}$. Historically, the first example of a non-Kähler symplectic 4-manifold, due to Thurston Th, is a non-trivial $T^{2}$-bundle over $T^{2}$ (the product of $S^{1}$ with the mapping torus of a Dehn twist, which has $b_{1}=3$ ).

Unfortunately, not many four-manifolds are fibered.

Definition 2.2. A Lefschetz pencil on a smooth oriented four-manifold $X$ is a map $f: X-\left\{b_{1}, \ldots, b_{n}\right\} \rightarrow S^{2}$, submersive away from a finite set $\left\{p_{1}, \ldots, p_{r}\right\}$, conforming to local models $(i)\left(z_{1}, z_{2}\right) \mapsto z_{1} / z_{2}$ near each $b_{i},($ ii $)\left(z_{1}, z_{2}\right) \mapsto z_{1}^{2}+z_{2}^{2}$ near each $p_{j}$. Here the $z_{i}$ are orientation-preserving local complex-valued coordinates.

We can additionally require that the critical values of $f$ are all distinct (so that each fiber contains at most one singular point).

This definition is motivated by the complex analogue of Morse theory. Global holomorphic functions on a projective surface must be constant, but interesting functions exist on the complement of finitely many points, and the generic such will have only quadratic singularities. The (closures of the) fibers of the map $f$ cut the four-manifold $X$ into a family of real surfaces all passing through the $b_{i}$ 
(locally like complex lines through a point in $\mathbb{C}^{2}$ ), and with certain fibers having nodal singularities $\left(\left(z_{1}+i z_{2}\right)\left(z_{1}-i z_{2}\right)=0\right)$. If we blow up the $b_{i}$, then the map $f$ extends to the entire manifold and we obtain a Lefschetz fibration.

A small generalization of the previous argument to the case of Lefschetz fibrations shows that, if $X$ admits a Lefschetz pencil, then it is symplectic (work on the blowup and choose the constant $C$ so large that the exceptional sections arising from the $b_{i}$ are all symplectic and can be symplectically blown down). In fact the symplectic form obtained in this way is canonical up to isotopy rather than just deformation equivalence, as shown by Gompf GS, Go2.

A real Morse function encodes the topology of a manifold: the critical values disconnect $\mathbb{R}$, and the topology of the level sets changes by a handle addition as we cross a critical value. In the complex case, the critical values do not disconnect, but the local model is determined by its monodromy, i.e. the diffeomorphism of the smooth fiber obtained by restricting the fibration to a circle enclosing a single critical value.

The fiber $F_{t}$ of the map $\left(z_{1}, z_{2}\right) \rightarrow z_{1}^{2}+z_{2}^{2}$ above $t \in \mathbb{C}$ is given by the equation $\left(z_{1}+i z_{2}\right)\left(z_{1}-i z_{2}\right)=t$ : the fiber $F_{t}$ is smooth (topologically an annulus) for all $t \neq 0$, while the fiber above the origin presents a transverse double point, and is obtained from the nearby fibers by collapsing an embedded simple closed loop called the vanishing cycle. For example, for $t>0$ the vanishing cycle is the loop $\left\{\left(x_{1}, x_{2}\right) \in \mathbb{R}^{2}, x_{1}^{2}+x_{2}^{2}=t\right\}=F_{t} \cap \mathbb{R}^{2} \subset F_{t}$.

Proposition 2.3. For a circle in the base $S^{2}$ of a Lefschetz fibration enclosing a single critical value, whose critical fiber has a single node, the monodromy is a Dehn twist about the vanishing cycle.

Sketch of proof. By introducing a cutoff function $\psi$ and by identifying the fiber $z_{1}^{2}+z_{2}^{2}=t$ with the set $z_{1}^{2}+z_{2}^{2}=\psi\left(\|z\|^{2}\right) t$, we can see that the monodromy is the identity outside a small neighborhood of the vanishing cycle. This reduces the problem to the local model of the annulus, which has mapping class group (relative to the boundary) isomorphic to $\mathbb{Z}$, so we just need to find one integer. One possibility is to study an example, e.g. an elliptic surface, where we can determine the monodromy by considering its action on homology, interpreted as periods. Alternatively, we can think of the annulus as a double cover of the disc branched at two points (the two square roots of $t$ ), and watch these move as $t$ follows the unit circle.
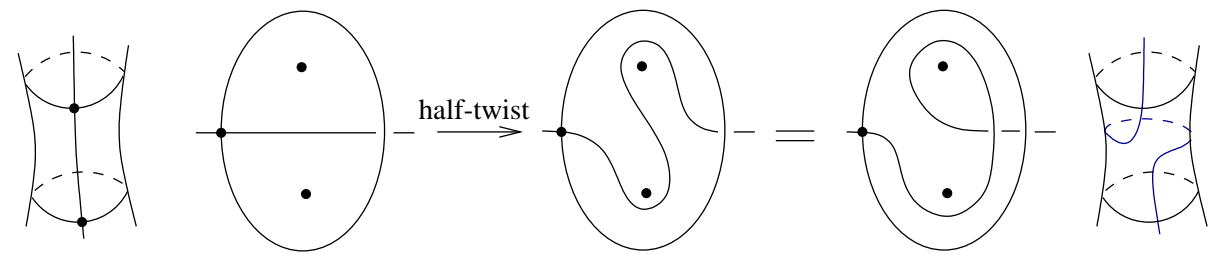

One can also consider higher-dimensional Lefschetz fibrations, which by definition are again submersions away from non-degenerate quadratic singularities. In the local model, the smooth fibers of $f:\left(z_{i}\right) \mapsto \sum z_{i}^{2}$ also contain a (Lagrangian) sphere, and the monodromy around the critical fiber $f^{-1}(0)$ is a generalized Dehn twist about this vanishing cycle (see the lectures by Seidel in this volume).

Using this local model, we may now define the monodromy homomorphism. Fix a base point $q_{0} \in S^{2}-\operatorname{crit}(f)$, and consider a closed loop $\gamma:[0,1] \rightarrow S^{2}-\operatorname{crit}(f)$ 
(starting and ending at $q_{0}$ ). By fixing a horizontal distribution we can perform parallel transport in the fibers of $f$ along $\gamma$, which induces a diffeomorphism from $\Sigma=f^{-1}\left(q_{0}\right)$ to itself. (Such a horizontal distribution is canonically defined if we fix a symplectic form on the total space by taking the symplectic orthogonal complements to the fibers.) The isotopy class of this diffeomorphism, which is welldefined independently of the chosen horizontal distribution, is called the monodromy of $f$ along $\gamma$. Hence, we obtain a monodromy homomorphism characteristic of the Lefschetz fibration $f$,

$$
\psi: \pi_{1}\left(S^{2}-\operatorname{crit}(f), q_{0}\right) \rightarrow \pi_{0} \operatorname{Diff}^{+}(\Sigma),
$$

which takes a loop encircling one critical value to a Dehn twist as above.

Example: let $C_{0}$ and $C_{1}$ be generic cubic curves in $\mathbb{C P}^{2}$, and consider the pencil of cubics $\left\{C_{0}+\lambda C_{1}=0\right\}_{\lambda \in \mathbb{C P}^{1}}$. This pencil has 9 base points (the intersections of $C_{0}$ and $C_{1}$ ), and 12 singular fibers. To see the latter fact, note that the Euler characteristic $\chi(X)$ of the total space of a Lefschetz pencil of genus $g$ is given by $\chi(X)=4-4 g-\# b_{i}+\# p_{j}$.

After blowing up the base points, we obtain an elliptic Lefschetz fibration, whose monodromy takes values in the genus 1 mapping class group $\operatorname{Map}_{1}=\pi_{0} \operatorname{Diff}^{+}\left(T^{2}\right)=$ $S L(2, \mathbb{Z})$. Each local monodromy is conjugate to $\left(\begin{array}{ll}1 & 1 \\ 0 & 1\end{array}\right)$. The monodromy homomorphism is determined by the images of a basis for $\pi_{1}\left(S^{2}-\operatorname{crit}(f)\right)$ consisting of 12 loops encircling one critical value each. The monodromy around the product of these loops is the identity, as the product loop bounds a disc in $S^{2}$ over which the fibration is trivial. In an appropriate basis, the resulting word in 12 Dehn twists in $S L(2, \mathbb{Z})$ can be brought into standard form

$$
(A \cdot B)^{6}=\left(\left(\begin{array}{ll}
1 & 1 \\
0 & 1
\end{array}\right) \cdot\left(\begin{array}{rr}
1 & 0 \\
-1 & 1
\end{array}\right)\right)^{6}=I .
$$

Such a word in Dehn twists, called a positive relation in the relevant mapping class group, captures the topology of a Lefschetz fibration. In the case where the fibration admits distinguished sections, e.g. coming from the base points of a pencil, we can refine the monodromy by working in the relative mapping class group of the pair $\left(\Sigma,\left\{b_{i}\right\}\right)$ (see the example in the last lecture).

In fact, a careful analysis of positive relations in $S L(2, \mathbb{Z})$ implies that all elliptic Lefschetz fibrations are Kähler (and have monodromy words of the form $(A \cdot B)^{6 n}=$ 1 ), a classical result of Moishezon and Livne Mo1. More geometrically, this result can also be deduced from the work of Siebert and Tian [ST] described in their lectures in this volume.

Remark. In the case of Lefschetz fibrations over a disc, the monodromy homomorphism determines the total space of the fibration up to symplectic deformation. When considering fibrations over $S^{2}$, the monodromy data determines the fibration over a large disc $D$ containing all critical values, after which we only need to add a trivial fibration over a small disc $D^{\prime}=S^{2}-D$, to be glued in a manner compatible with the fibration structure over the common boundary $\partial D=\partial D^{\prime}=S^{1}$. This gluing involves the choice of a map from $S^{1}$ to $\operatorname{Diff}^{+}\left(\Sigma_{g}\right)$, i.e. an element of $\pi_{1} \operatorname{Diff}^{+}\left(\Sigma_{g}\right)$, which is trivial if $g \geq 2$ (hence in this case the positive relation determines the topology completely). 
Combining the facts that Lefschetz pencils carry symplectic structures and that they correspond to positive relations has algebraic consequences for the mapping class group.

Proposition 2.4. There is no positive relation involving only Dehn twists about separating curves.

Sketch of proof. (see Sm1 for a harder proof) - Suppose for contradiction we have such a word of length $\delta$. This defines a four-manifold $X$ with a Lefschetz fibration having this as monodromy. We can compute the signature $\sigma(X)$ by surgery, cutting the manifold open along neighborhoods of the singular fibers; we find (cf. $\mathrm{Oz}$ ) that each local model contributes -1 to the signature, so we obtain $\sigma(X)=-\delta$. This allows us to compute the Betti and Chern numbers of $X: b_{1}(X)=2 g, b_{2}(X)=\delta+2$, $c_{2}(X)=4-4 g+\delta$, and $c_{1}^{2}(X)=8-8 g-\delta<-\delta$. Hence $c_{1}^{2}$ of any minimal model of $X$ is negative, and so $X$ must be rational or ruled by a theorem of Liu. These cases can be excluded by hand.

The pencil of cubics on $\mathbb{C P}^{2}$ is an instance of a much more general construction.

Proposition 2.5 (Lefschetz). Projective surfaces have Lefschetz pencils.

Generic hyperplane sections cut out smooth complex curves, and a pencil corresponds to a line of such hyperplanes. Inside the dual projective space $\left(\mathbb{C P}^{N}\right)^{*}$ pick a line transverse to the dual variety (the locus of hyperplanes which are not transverse to the given surface). A local computation shows that this transversality condition goes over to give exactly the non-degenerate critical points of a Lefschetz pencil. From another point of view, if $L$ is a very ample line bundle on $X$ (so sections generate the fibers of $L$ ), we can consider the evaluation map $X \times H^{0}(L) \rightarrow L$. Let $Z \subset X \times H^{0}(L)$ be the preimage of the zero section, then a regular value of the projection $Z \rightarrow H^{0}(L)$ is a section with smooth zero set. In this way the construction of embedded complex curves in $X$ (and more generally linear systems of such) can be reduced to the existence of regular values, i.e. Sard's theorem.

Certainly the converse to the above cannot be true: not all Lefschetz fibrations are Kähler. The easiest way to see this is to use the (twisted) fiber sum construction. Given a positive relation $\tau_{1} \ldots \tau_{s}=1$ in $\operatorname{Map}_{g}$, and some element $\phi \in \operatorname{Map}_{g}$, we obtain a new positive relation $\tau_{1} \ldots \tau_{s}\left(\phi^{-1} \tau_{1} \phi\right) \ldots\left(\phi^{-1} \tau_{s} \phi\right)=1$. If $\phi=$ Id the corresponding four-manifold is the double branched cover of the original manifold over a union of two smooth fibers, but in general the operation has no holomorphic interpretation. The vanishing cycles of the new fibration are the union of the old vanishing cycles and their images under $\phi$. Since $H_{1}(X)=H_{1}(\Sigma) /\langle$ vanishing cycles , we can easily construct examples with odd first Betti number, for example starting with a genus 2 pencil on $T^{2} \times S^{2}$ OS.

More sophisticated examples (for instance with trivial first Betti number) can be obtained by forming infinite families of twisted fiber sums with non-conjugate monodromy groups and invoking the following

Theorem 2.6 (Arakelov-Paršin). Only finitely many isotopy classes of Lefschetz fibrations with given fiber genus and number of critical fibers can be Kähler.

Remark. Twisted fiber sum constructions can often be "untwisted" by subsequently fiber summing with another suitable (e.g. holomorphic) Lefschetz fibration. A consequence of this is a stable isotopy result for genus 2 Lefschetz fibrations [Au6]: 
any genus 2 Lefschetz fibration becomes isotopic to a holomorphic fibration after repeated fiber sums with the standard holomorphic fibration with 20 singular fibers coming from a genus 2 pencil on $\mathbb{C P}^{1} \times \mathbb{C P}^{1}$. More generally a similar result holds for all Lefschetz fibrations with monodromy contained in the hyperelliptic subgroup of the mapping class group. This is a corollary of a recent result of Kharlamov and Kulikov [KK] about braid monodromy factorizations: after repeated (untwisted) fiber sums with copies of a same fixed holomorphic fibration with $8 g+4$ singular fibers, any hyperelliptic genus $g$ Lefschetz fibration eventually becomes holomorphic. Moreover, the fibration obtained in this manner is completely determined by its number of singular fibers of each type (irreducible, reducible with components of given genus), and when the fiber genus is odd by a certain $\mathbb{Z}_{2}$-valued invariant. (The proof of this result uses the fact that the hyperelliptic mapping class group is an extension by $\mathbb{Z}_{2}$ of the braid group of $2 g+2$ points on a sphere, which is itself a quotient of $B_{2 g+2}$; this makes it possible to transform the monodromy of a hyperelliptic Lefschetz fibration into a factorization in $B_{2 g+2}$, with different types of factors for the various types of singular fibers and extra contributions belonging to the kernel of the morphism $B_{2 g+2} \rightarrow B_{2 g+2}\left(S^{2}\right)$, and hence reduce the problem to that studied by Kharlamov and Kulikov. This connection between mapping class groups and braid groups will be further studied in later lectures.) It is not clear whether the result should be expected to remain true in the non-hyperelliptic case.

These examples of Lefschetz fibrations differ somewhat in character from those obtained in projective geometry, since the latter always admit exceptional sections of square -1 . However, an elementary argument in hyperbolic geometry shows that fiber sums never have this property [Sm3]. To see that not every Lefschetz pencil arises from a holomorphic family of surfaces appears to require some strictly deeper machinery.

To introduce this, let us note that, if we choose a metric on the total space of a genus $g$ Lefschetz pencil or fibration, the fibers become Riemann surfaces and this induces a map $\phi: \mathbb{C P}^{1} \rightarrow \overline{\mathcal{M}}_{g}$ to the Deligne-Mumford moduli space of stable genus $g$ curves. There is a line bundle $\lambda \rightarrow \overline{\mathcal{M}}_{g}$ (the Hodge bundle), with fiber $\operatorname{det} H^{0}\left(K_{\Sigma}\right)$ above $[\Sigma]$, and an index theorem for the family of $\bar{\partial}$-operators on the fibers shows

Proposition 2.7 ([Sm1] $) . \sigma(X)=4\left\langle c_{1}(\lambda),\left[\phi\left(\mathbb{C P}^{1}\right)\right]\right\rangle-\delta$.

On the other hand, $c_{1}(\lambda)$ and the Poincaré duals of the components of the divisor of nodal curves generate $H^{2}\left(\overline{\mathcal{M}}_{g}, \mathbb{Z}\right)$, so the above formula - together with the numbers of singular fibers of different topological types - characterizes the homology class $\left[\phi\left(\mathbb{C P}^{1}\right)\right]$.

Clearly, holomorphic Lefschetz fibrations give rise to rational curves in the moduli space, and these have locally positive intersection with all divisors in which they are not contained. This gives another constraint on which Lefschetz pencils and fibrations can be holomorphic. For example, the divisor $\overline{\mathcal{H}}_{3}$ of hyperelliptic genus 3 curves has homology class $\left[\overline{\mathcal{H}}_{3}\right]=9 c_{1}(\lambda)-\left[\Delta_{0}\right]-3\left[\Delta_{1}\right]$, where $\Delta_{0}$ and $\Delta_{1}$ are the divisors of irreducible and reducible nodal curves respectively.

Corollary 2.8. A genus 3 Lefschetz fibration $X$ with irreducible fibers and such that (i) $\chi(X)+1$ is not divisible by 7 , (ii) $9 \sigma(X)+5 \chi(X)+40<0$ is not holomorphic. 
Sketch of proof. For hyperelliptic fibrations of any genus, we have

$$
(8 g+4) c_{1}(\lambda) \cdot\left[\phi\left(S^{2}\right)\right]=g\left[\Delta_{0}\right] \cdot\left[\phi\left(S^{2}\right)\right]+\sum_{h=1}^{[g / 2]} 4 h(g-h)\left[\Delta_{h}\right] \cdot\left[\phi\left(S^{2}\right)\right] .
$$

To prove this, we can represent the four-manifold as a double cover of a rational ruled surface (see the lectures of Siebert and Tian in this volume). This gives another expression for $\sigma(X)$ which can be compared to that above. Then assumption $(i)$ and integrality of the signature show that the fibration is not isotopic to a hyperelliptic fibration, while assumption $(i i)$ shows that $\left[\phi\left(S^{2}\right)\right] \cdot\left[\overline{\mathcal{H}}_{3}\right]<0$.

It is possible to build a Lefschetz fibration (with 74 singular fibers) admitting a $(-1)$-section and satisfying the conditions of the Corollary [Sm2].

The right correspondence between the geometry and the algebra comes from the work of Donaldson [Do2, Do3]:

Theorem 2.9 (Donaldson). Any compact symplectic 4-manifold admits Lefschetz pencils with symplectic fibers (if $[\omega]$ is integral, Poincaré dual to $k[\omega]$ for any sufficiently large $k$ ).

As explained later, we even get some uniqueness - but only asymptotically with the parameter $k$. Increasing the parameter $k$ makes the algebraic monodromy descriptions more and more complicated, but in principle, as with surgery theory for high-dimensional smooth manifolds, this gives a complete algebraic encoding. The construction is flexible enough to impose some extra conditions on the pencils, for instance we can assume that all the singular fibers are irreducible. (If the four-manifold has even intersection form, this is completely elementary.)

In order to describe Donaldson's construction of symplectic Lefschetz pencils, we need a digression into approximately holomorphic geometry.

2.2. Approximately holomorphic geometry. On an almost-complex manifold, the lack of integrability usually prevents the existence of non-trivial holomorphic sections of vector bundles or pseudo-holomorphic maps to other manifolds, but one can work in a similar manner with approximately holomorphic objects.

Let $\left(M^{2 n}, \omega\right)$ be a compact symplectic manifold of dimension $2 n$. We will assume throughout this paragraph that $\frac{1}{2 \pi}[\omega] \in H^{2}(M, \mathbb{Z})$; this integrality condition does not restrict the topological type of $M$, since any symplectic form can be perturbed into another symplectic form $\omega^{\prime}$ whose cohomology class is rational (we can then achieve integrality by multiplication by a constant factor). Morever, it is easy to check that the submanifolds of $M$ that we will construct are not only $\omega^{\prime}$-symplectic but also $\omega$-symplectic, hence making the general case of Theorem 2.9 follow from the integral case.

Let $J$ be an almost-complex structure compatible with $\omega$, and let $g(.,)=.\omega(., J$. be the corresponding Riemannian metric. We consider a complex line bundle $L$ over $M$ such that $c_{1}(L)=\frac{1}{2 \pi}[\omega]$, endowed with a Hermitian metric and a Hermitian connection $\nabla^{L}$ with curvature 2 -form $F\left(\nabla^{L}\right)=-i \omega$. The almost-complex structure induces a splitting of the connection : $\nabla^{L}=\partial^{L}+\bar{\partial}^{L}$, where $\partial^{L} s(v)=\frac{1}{2}\left(\nabla^{L} s(v)-\right.$ $\left.i \nabla^{L} s(J v)\right)$ and $\bar{\partial}^{L} s(v)=\frac{1}{2}\left(\nabla^{L} s(v)+i \nabla^{L} s(J v)\right)$.

If the almost-complex structure $J$ is integrable, i.e. if $M$ is Kähler, then $L$ is an ample holomorphic line bundle, and for large enough values of $k$ the holomorphic sections of $L^{\otimes k}$ determine an embedding of the manifold $M$ into a projective space 
(Kodaira's theorem). Generic hyperplane sections of this projective embedding are smooth hypersurfaces in $M$, and a pencil of hyperplanes through a generic codimension 2 linear subspace defines a Lefschetz pencil.

When the manifold $M$ is only symplectic, the lack of integrability of $J$ prevents the existence of holomorphic sections. Nonetheless, it is possible to find an approximately holomorphic local model: a neighborhood of a point $x \in M$, equipped with the symplectic form $\omega$ and the almost-complex structure $J$, can be identified with a neighborhood of the origin in $\mathbb{C}^{n}$ equipped with the standard symplectic form $\omega_{0}$ and an almost-complex structure of the form $i+O(|z|)$. In this local model, the line bundle $L^{\otimes k}$ endowed with the connection $\nabla=\left(\nabla^{L}\right)^{\otimes k}$ of curvature $-i k \omega$ can be identified with the trivial line bundle $\mathbb{C}$ endowed with the connection $d+\frac{k}{4} \sum\left(z_{j} d \bar{z}_{j}-\bar{z}_{j} d z_{j}\right)$. The section of $L^{\otimes k}$ given in this trivialization by $s_{\text {ref }, k, x}(z)=\exp \left(-\frac{1}{4} k|z|^{2}\right)$ is then approximately holomorphic Do1.

More precisely, a sequence of sections $s_{k}$ of $L^{\otimes k}$ is said to be approximately holomorphic if, with respect to the rescaled metrics $g_{k}=k g$, and after normalization of the sections to ensure that $\left\|s_{k}\right\|_{C^{r}, g_{k}} \sim C$, an inequality of the form $\left\|\bar{\partial} s_{k}\right\|_{C^{r-1}, g_{k}}<C^{\prime} k^{-1 / 2}$ holds, where $C$ and $C^{\prime}$ are constants independent of $k$. The change of metric, which dilates all distances by a factor of $\sqrt{k}$, is required in order to be able to obtain uniform estimates, due to the large curvature of the line bundle $L^{\otimes k}$. The intuitive idea is that, for large $k$, the sections of the line bundle $L^{\otimes k}$ with curvature $-i k \omega$ probe the geometry of $M$ at small scale $(\sim 1 / \sqrt{k})$, which makes the almost-complex structure $J$ almost integrable and allows one to achieve better approximations of the holomorphicity condition $\bar{\partial} s=0$.

Since the above requirement is an open condition, there is no well-defined "space of approximately holomorphic sections" of $L^{\otimes k}$. Nonetheless, the above local model gives us a large number of approximately holomorphic sections (consider $s_{\text {ref, } k, x}$ for a large finite set of $x \in X$ ), which can be used to embed $X$ as a symplectic submanifold of a (high-dimensional) projective space. However, this embedding by itself is not very useful since it is not clear that any of its hyperplane sections can be used to define a smooth symplectic hypersurface in $X$.

Hence, in contrast with the complex case, the non-trivial part of the construction is to find, among all the available approximately holomorphic sections, some whose geometric behavior is as generic as possible. That this is at all possible is a subtle observation of Donaldson, which leads to the following result [Do1]:

Theorem 2.10 (Donaldson). For $k \gg 0, L^{\otimes k}$ admits approximately holomorphic sections $s_{k}$ whose zero sets $W_{k}$ are smooth symplectic hypersurfaces.

The proof of this result starts from the observation that, if the section $s_{k}$ vanishes transversely and if $\left|\bar{\partial} s_{k}(x)\right| \ll\left|\partial s_{k}(x)\right|$ at every point of $W_{k}=s_{k}^{-1}(0)$, then the submanifold $W_{k}$ is symplectic, and even approximately $J$-holomorphic (i.e. $J\left(T W_{k}\right)$ is close to $T W_{k}$ ). The crucial point is therefore to obtain a lower bound for $\partial s_{k}$ at every point of $W_{k}$, in order to make up for the lack of holomorphicity.

Sections $s_{k}$ of $L^{\otimes k}$ are said to be uniformly transverse to 0 if there exists a constant $\eta>0$ (independent of $k$ ) such that the inequality $\left|\partial s_{k}(x)\right|_{g_{k}}>\eta$ holds at any point of $M$ where $\left|s_{k}(x)\right|<\eta$. In order to prove Theorem [2.10] it is sufficient to achieve this uniform estimate on the tranversality of some approximately holomorphic sections $s_{k}$. The idea of the construction of such sections consists of 
two main steps. The first one is an effective local transversality result for complexvalued functions, for which Donaldson's argument appeals to ideas of Yomdin about the complexity of real semi-algebraic sets (see Aus for a simplification of the argument). The second step is a remarkable globalization process, which makes it possible to achieve uniform transversality over larger and larger open subsets by means of successive perturbations of the sections $s_{k}$, until transversality holds over the entire manifold $M$ [Do1].

That the interplay between the two steps above is subtle can already be gathered from the delicate statement of the local transversality result. For $\beta>0$ set $t(\beta)=$ $\beta /\left(\log \beta^{-1}\right)^{d}$. Here $\beta$ represents the maximum size of the allowed perturbation $s_{\text {given }} \mapsto s_{\text {given }}-w s_{\text {ref }}$ with $|w|<\beta, t(\beta)$ is the amount of transversality thereby obtained, and $d=d(n)$ is a universal constant that we will mostly ignore. Write $B^{+} \subset \mathbb{C}^{n}$ for a Euclidean ball slightly larger than the unit ball $B$.

Theorem 2.11 ([Do1, $\overline{\mathrm{Au} 2}]$ ). If $f: B^{+} \rightarrow \mathbb{C}^{n+1}$ satisfies $|f|<1$ and $|\bar{\partial} f|<t(\beta)$ pointwise, then there is some $w \in \mathbb{C}^{n+1}$ with $|w|<\beta$ such that $|f(z)-w|>t(\beta)$ over $B \subset B^{+}$.

To see the relevance of this, for an approximately holomorphic section $s_{k}$ of $L^{\otimes k}$ we consider the holomorphic 1-jet $\left(s_{k}, \partial s_{k}\right)$, a section of $L^{\otimes k} \oplus L^{\otimes k} \otimes T^{*} M^{1,0}$. This is locally a map from the complex $n$-dimensional ball to $\mathbb{C}^{n+1}$, and by adding reference sections we can explicitly give local perturbations of this. In this context, theorems such as the one above are elementary if we take a polynomial function $t(\beta)=($ const $) \beta^{q}$ with $q>1$, but such perturbations do not patch well.

By way of an example, let $f: \mathbb{R}^{4} \rightarrow \mathbb{R}^{6}$ have bounded derivative, so $f$ takes balls of radius $\epsilon$ to balls of comparable size. Now $B^{4}(1)$ is filled by approximately $\epsilon^{-4}$ balls, and each is taken to a ball of volume approximately $\epsilon^{6}$. Hence, the total volume of the image is about $\epsilon^{2}$ of $B^{6}(1)$. To change the function $f \mapsto f-w$ by $\beta$ in order to miss the image of $f$ by $t(\beta)=($ const $) \beta^{3}$, say, would be straightforward, because taking $\epsilon=t(\beta)$, the $\epsilon$-neighborhood of $f\left(B^{4}\right)$ has a volume of the order of $\epsilon^{2}$ and hence cannot contain any ball of radius $\epsilon^{1 / 3} \sim \beta$.

However, our manifold is covered by $O\left(k^{2 n}\right)$ balls of fixed $g_{k}$-radius in which our reference sections $s_{\text {ref, } k}$ are concentrated. Perturbing over each ball one by one, all estimates are destroyed and it is impossible to achieve uniform transversality. The solution is to perturb over balls at great distance simultaneously; nonetheless the simultaneous perturbations will not be entirely independent. We cover $X$ by a fixed number $D^{2 n}$ of collections of balls (the number of balls in each collection, but not the number of collections, will grow with the parameter $k$ ), in such a way that any two balls in the same collection are at $g_{k}$-distance at least $D$ from each other. To obtain uniformly transverse sections, we (1) start with some approximately holomorphic section (e.g. the zero section $s_{0}=0$ ); (2) perturb by $\beta_{0}$ over balls of the first collection $I_{0}$ to get a section $s_{1}$ which is $t\left(\beta_{0}\right)$ transverse over $\cup_{i \in I_{0}} B_{i}$; (3) perturb over balls of the second collection $I_{1}$ by an amount $\beta_{1} \ll t\left(\beta_{0}\right) / 2$ to get a section $s_{2}$ which is $t\left(\beta_{1}\right)$-transverse over $\cup_{i \in I_{0} \cup I_{1}} B_{i}$ etc. Continuing, we need the sequence

$$
\beta_{0}, \beta_{1} \sim t\left(\beta_{0}\right), \beta_{2} \sim t\left(\beta_{1}\right) \ldots
$$

to be chosen so that, at each stage (for all $\left.N<D^{2 n}\right)$, $\exp \left(-D^{2} \beta_{N}\right)<\beta_{N+1} / 2$. Here, the left-hand side $e^{-D^{2}} \beta_{N}$ is the effect of the perturbation at a ball $B_{1}$ in the collection $I_{N}$ on another ball $B_{2}$ of the same collection (which we perturb 
simultaneously); the right-hand side $\beta_{N+1} / 2$ is the transversality obtained at the ball $B_{2}$ by virtue of its own perturbation. We have $N=D^{2 n}$ stages, for some large $D$, so we need

$$
\exp \left(-N^{1 / n}\right) \beta_{N}<\beta_{N+1} / 2
$$

for $N \gg 0$. This inequality fails for any polynomial function $\beta_{N+1}=t\left(\beta_{N}\right)=\beta_{N}^{q} / 2$ for $q>1$ : one gets $\beta_{N} \sim(1 / 2)^{q^{N}}$ and $\exp \left(-N^{1 / n}\right) \nless \beta_{N+1} / \beta_{N}$.

In other words, the estimates coming naively from Sard's theorem don't provide a good enough local theorem to pass to a global one. The remedy is that our functions are approximately holomorphic and not arbitrary, and for holomorphic functions stronger Sard-like theorems are available: the prototype here is that the regular values of a smooth map $B(1) \subset \mathbb{R}^{2 n} \rightarrow \mathbb{R}^{2}$ are in general only dense, but the regular values of a holomorphic map $B(1) \subset \mathbb{C}^{n} \rightarrow \mathbb{C}$ form the complement of a finite set. In practice, the proof of Theorem 2.11 proceeds by reduction to the case of polynomial functions, and then appeals either to real algebraic geometry Do1] or to the classical monotonicity theorem $\mathrm{Au} 5$.

The symplectic submanifolds constructed by Donaldson present several remarkable properties which make them closer to complex submanifolds than to arbitrary symplectic submanifolds. For instance, they satisfy the Lefschetz hyperplane theorem: up to half the dimension of the submanifold, the homology and homotopy groups of $W_{k}$ are identical to those of $M$ [Do1. More importantly, these submanifolds are, in a sense, asymptotically unique: for given large enough $k$, the submanifolds $W_{k}$ are, up to symplectic isotopy, independent of all the choices made in the construction (including that of the almost-complex structure $J$ ) Au1].

It is worth mentioning that analogues of this construction have been obtained for contact manifolds by Ibort, Martinez-Torres and Presas (IMP, ...); see also recent work of Giroux and Mohsen Gi].

As an application of this theorem, we mention a symplectic packing result due to Biran [Bi]. Recall that a full symplectic packing of a manifold $(M, \omega)$ is an embedding $\left(\amalg B_{i}, \omega_{s t d}\right) \hookrightarrow(M, \omega)$ of a disjoint union of standard Euclidean symplectic balls of equal volumes whose images fill the entire volume of $M$. Gromov pointed out that, in contrast to the volume-preserving case, there are obstructions to symplectic packing: for instance, $\mathbb{C P}^{2}$ cannot be fully packed by two balls.

Theorem 2.12 (Biran). Let $\left(M^{4}, \omega\right)$ be a symplectic four-manifold with integral $[\omega]$. Then $M$ admits a full packing by $N$ balls for all large $N$.

The key ingredient in the proof is to reduce to the case of ruled surfaces by decomposing $M$ into a disc bundle over a Donaldson submanifold $\Sigma$ dual to $k[\omega]$ and an isotropic CW-complex - which takes no volume. It is therefore sufficient to fully pack a ruled surface by balls which remain disjoint from a section at infinity. On the other hand, there is a well-known correspondence between embeddings of symplectic balls of size $\mu$ and symplectic forms on a blow-up giving each exceptional curve area $\mu$. Moreover, symplectic forms in a given cohomology class can be constructed by symplectic inflation in the presence of appropriate embedded symplectic surfaces, and for ruled surfaces these are provided by an elementary computation of the Gromov or Seiberg-Witten invariants. The best value of $N$ is not known in general, because it is determined by the best value of $k$ in Donaldson's construction.

We now move on to Donaldson's construction of symplectic Lefschetz pencils Do2, Do3]. In comparison with Theorem 2.10 the general setup is the same, the 
main difference being that we consider no longer one, but two sections of $L^{\otimes k}$. A pair of suitably chosen approximately holomorphic sections $\left(s_{k}^{0}, s_{k}^{1}\right)$ of $L^{\otimes k}$ defines a family of symplectic hypersurfaces

$$
\Sigma_{k, \alpha}=\left\{x \in M, s_{k}^{0}(x)-\alpha s_{k}^{1}(x)=0\right\}, \quad \alpha \in \mathbb{C P}^{1}=\mathbb{C} \cup\{\infty\} .
$$

The submanifolds $\Sigma_{k, \alpha}$ are all smooth except for finitely many of them which present an isolated singularity; they intersect transversely along the base points of the pencil, which form a smooth symplectic submanifold $Z_{k}=\left\{s_{k}^{0}=s_{k}^{1}=0\right\}$ of codimension 4.

The two sections $s_{k}^{0}$ and $s_{k}^{1}$ determine a projective map $f_{k}=\left(s_{k}^{0}: s_{k}^{1}\right): M-Z_{k} \rightarrow$ $\mathbb{C P}^{1}$, whose critical points correspond to the singularities of the fibers $\Sigma_{k, \alpha}$. In the case of a symplectic Lefschetz pencil, the function $f_{k}$ is a complex Morse function, i.e. near any of its critical points it is given by the local model $f_{k}(z)=z_{1}^{2}+\cdots+z_{n}^{2}$ in approximately holomorphic coordinates. After blowing up $M$ along $Z_{k}$, the Lefschetz pencil structure on $M$ gives rise to a well-defined map $\hat{f}_{k}: \hat{M} \rightarrow \mathbb{C P}^{1}$; this map is a symplectic Lefschetz fibration. Hence, Theorem 2.9 may be reformulated more precisely as follows:

Theorem 2.13 (Donaldson). For large enough $k$, the given manifold $\left(M^{2 n}, \omega\right)$ admits symplectic Lefschetz pencil structures determined by pairs of suitably chosen approximately holomorphic sections $s_{k}^{0}, s_{k}^{1}$ of $L^{\otimes k}$. Moreover, for large enough $k$ these Lefschetz pencil structures are uniquely determined up to isotopy.

As in the case of submanifolds, Donaldson's argument relies on successive perturbations of given approximately holomorphic sections $s_{k}^{0}$ and $s_{k}^{1}$ in order to achieve uniform transversality properties, not only for the sections $\left(s_{k}^{0}, s_{k}^{1}\right)$ themselves but also for the derivative $\partial f_{k}$ Do3.

The precise meaning of the uniqueness statement is the following: assume we are given two sequences of Lefschetz pencil structures on $(M, \omega)$, determined by pairs of approximately holomorphic sections of $L^{\otimes k}$ satisfying uniform transversality estimates, but possibly with respect to two different $\omega$-compatible almost-complex structures on $M$. Then, beyond a certain (non-explicit) value of $k$, it becomes possible to find one-parameter families of Lefschetz pencil structures interpolating between the given ones. In particular, this implies that for large $k$ the monodromy invariants associated to these Lefschetz pencils only depend on $(M, \omega, k)$ and not on the choices made in the construction.

The monodromy invariants associated to a symplectic Lefschetz pencil are essentially those of the symplectic Lefschetz fibration obtained after blow-up along the base points, with only a small refinement. After the blow-up operation, each fiber of $\hat{f}_{k}: \hat{M} \rightarrow \mathbb{C P}^{1}$ contains a copy of the base locus $Z_{k}$ embedded as a smooth symplectic hypersurface. This hypersurface lies away from all vanishing cycles, and is preserved by the monodromy. Hence, the monodromy homomorphism can be defined to take values in the group of isotopy classes of symplectomorphisms of the fiber $\Sigma_{k}$ whose restriction to the submanifold $Z_{k}$ is the identity.

\section{Symplectic BRAnChed COVERS OF $\mathbb{C P}^{2}$}

\subsection{Symplectic branched covers.}

Definition 3.1. A smooth map $f: X^{4} \rightarrow\left(Y^{4}, \omega_{Y}\right)$ from a compact oriented smooth 4-manifold to a compact symplectic 4-manifold is a (generic) symplectic branched 
covering if, given any point $p \in X$, there exist neighborhoods $U \ni p$ and $V \ni f(p)$ and orientation-preserving local diffeomorphisms $\phi: U \rightarrow \mathbb{C}^{2}$ and $\psi: V \rightarrow \mathbb{C}^{2}$, such that $\psi_{*} \omega_{Y}(v, i v)>0 \forall v \neq 0$ (i.e. the standard complex structure is $\psi_{*} \omega_{Y}$-tame), and such that $\psi \circ f \circ \phi^{-1}$ is one of the following model maps:

(i) $(u, v) \mapsto(u, v)$ (local diffeomorphism),

(ii) $(u, v) \mapsto\left(u^{2}, v\right)$ (simple branching),

(iii) $(u, v) \mapsto\left(u^{3}-u v, v\right)$ (cusp).

The three local models appearing in this definition are exactly those describing a generic holomorphic map between complex surfaces, except that the local coordinate systems we consider are not holomorphic.

By computing the Jacobian of $f$ in the given local coordinates, we can see that the ramification curve $R \subset X$ is a smooth submanifold (it is given by $\{u=0\}$ in the second local model and $\left\{v=3 u^{2}\right\}$ in the third one). However, the image $D=f(R) \subset X$ (the branch curve, or discriminant curve) may be singular. More precisely, in the simple branching model $D$ is given by $\left\{z_{1}=0\right\}$, while in the cusp model we have $f\left(u, 3 u^{2}\right)=\left(-2 u^{3}, 3 u^{2}\right)$, and hence $D$ is locally identified with the singular curve $\left\{27 z_{1}^{2}=4 z_{2}^{3}\right\} \subset \mathbb{C}^{2}$. This means that, at the cusp points, $D$ fails to be immersed. Besides the cusps, the branch curve $D$ also generically presents transverse double points (or nodes), which do not appear in the local models because they correspond to simple branching in two distinct points $p_{1}, p_{2}$ of the same fiber of $f$. There is no constraint on the orientation of the local intersection between the the two branches of $D$ at a node (positive or negative, i.e. complex or anti-complex), because the local models near $p_{1}$ and $p_{2}$ hold in different coordinate systems on $Y$.

Generically, the only singularities of the branch curve $D \subset Y$ are transverse double points ("nodes") of either orientation and complex cusps. Moreover, because the local models identify $D$ with a complex curve, the tameness condition on the coordinate systems implies that $D$ is a (singular) symplectic submanifold of $Y$.

The following result states that a symplectic branched cover of a symplectic 4-manifold carries a natural symplectic structure $\mathrm{Au2}$ :

Proposition 3.2. If $f: X^{4} \rightarrow\left(Y^{4}, \omega_{Y}\right)$ is a symplectic branched cover, then $X$ carries a symplectic form $\omega_{X}$ such that $\left[\omega_{X}\right]=f^{*}\left[\omega_{Y}\right]$, canonically determined up to symplectomorphism.

Proof. The 2-form $f^{*} \omega_{Y}$ is closed, but it is only non-degenerate outside of $R$. At any point $p$ of $R$, the 2-plane $K_{p}=\operatorname{Ker} d f_{p} \subset T_{p} X$ carries a natural orientation induced by the complex orientation in the local coordinates of Definition 3.1 Using the local models, we can construct an exact 2 -form $\alpha$ such that, at any point $p \in R$, the restriction of $\alpha$ to $K_{p}$ is non-degenerate and positive.

More precisely, given $p \in R$ we consider a small ball centered at $p$ and local coordinates $(u, v)$ such that $f$ is given by one of the models of the definition, and we set $\alpha_{p}=d\left(\chi_{1}(|u|) \chi_{2}(|v|) x d y\right)$, where $x=\operatorname{Re}(u), y=\operatorname{Im}(u)$, and $\chi_{1}$ and $\chi_{2}$ are suitably chosen smooth cut-off functions. We then define $\alpha$ to be the sum of these $\alpha_{p}$ when $p$ ranges over a finite subset of $R$ for which the supports of the $\alpha_{p}$ cover the entire ramification curve $R$. Since $f^{*} \omega_{Y} \wedge \alpha$ is positive at every point of $R$, it is easy to check that the 2 -form $\omega_{X}=f^{*} \omega_{Y}+\epsilon \alpha$ is symplectic for a small enough value of the constant $\epsilon>0$.

The fact that $\omega_{X}$ is canonical up to symplectomorphism follows immediately from Moser's stability theorem and from the observation that the space of exact 
perturbations $\alpha$ such that $\alpha_{\mid K_{p}}>0 \forall p \in R$ is a convex subset of $\Omega^{2}(X)$ and hence connected.

Approximately holomorphic techniques make it possible to show that every compact symplectic 4 -manifold can be realized as a branched cover of $\mathbb{C P}^{2}$. The general setup is similar to Donaldson's construction of symplectic Lefschetz pencils: we consider a compact symplectic manifold $(X, \omega)$, and perturbing the symplectic structure if necessary we may assume that $\frac{1}{2 \pi}[\omega] \in H^{2}(X, \mathbb{Z})$. Introducing an almost-complex structure $J$ and a line bundle $L$ with $c_{1}(L)=\frac{1}{2 \pi}[\omega]$, we consider triples of approximately holomorphic sections $\left(s_{k}^{0}, s_{k}^{1}, s_{k}^{2}\right)$ of $L^{\otimes k}$ : for $k \gg 0$, it is again possible to achieve a generic behavior for the projective map $f_{k}=\left(s_{k}^{0}: s_{k}^{1}: s_{k}^{2}\right): X \rightarrow \mathbb{C P}^{2}$ associated with the linear system. If the manifold $X$ is four-dimensional, then the linear system generically has no base points, and for a suitable choice of sections the map $f_{k}$ is a branched covering Au2.

Theorem 3.3. For large enough $k$, three suitably chosen approximately holomorphic sections of $L^{\otimes k}$ over $\left(X^{4}, \omega\right)$ determine a symplectic branched covering $f_{k}$ : $X^{4} \rightarrow \mathbb{C P}^{2}$, described in approximately holomorphic local coordinates by the local models of Definition 3.1. Moreover, for $k \gg 0$ these branched covering structures are uniquely determined up to isotopy.

Because the local models hold in approximately holomorphic (and hence $\omega$-tame) coordinates, the ramification curve $R_{k}$ of $f_{k}$ is a symplectic submanifold in $X$ (connected, since the Lefschetz hyperplane theorem applies). Moreover, if we normalize the Fubini-Study symplectic form on $\mathbb{C P}^{2}$ in such a way that $\frac{1}{2 \pi}\left[\omega_{F S}\right]$ is the generator of $H^{2}\left(\mathbb{C P}^{2}, \mathbb{Z}\right)$, then we have $\left[f_{k}^{*} \omega_{F S}\right]=2 \pi c_{1}\left(L^{\otimes k}=k[\omega]\right.$, and it is fairly easy to check that the symplectic form on $X$ obtained by applying Proposition 3.2 to the branched covering $f_{k}$ coincides up to symplectomorphism with $k \omega$ Au2. In fact, the exact 2-form $\alpha=k \omega-f_{k}^{*} \omega_{F S}$ is positive over Ker $d f_{k}$ at every point of $R_{k}$, and $f_{k}^{*} \omega_{F S}+t \alpha$ is a symplectic form for all $t \in(0,1]$.

The uniqueness statement in Theorem 3.3. which should be interpreted exactly in the same way as that obtained by Donaldson for Lefschetz pencils, implies that for $k \gg 0$ it is possible to define invariants of the symplectic manifold $(X, \omega)$ in terms of the monodromy of the branched covering $f_{k}$ and the topology of its branch curve $D_{k} \subset \mathbb{C P}^{2}$. However, the branch curve $D_{k}$ is only determined up to creation or cancellation of (admissible) pairs of nodes of opposite orientations.

A similar construction can be attempted when $\operatorname{dim} X>4$; in this case, the set of base points $Z_{k}=\left\{s_{k}^{0}=s_{k}^{1}=s_{k}^{2}=0\right\}$ is no longer empty; it is generically a smooth codimension 6 symplectic submanifold. With this understood, Theorem 3.3 admits the following higher-dimensional analogue $\mathrm{Au} 3$ :

Theorem 3.4. For large enough $k$, three suitably chosen approximately holomorphic sections of $L^{\otimes k}$ over $\left(X^{2 n}, \omega\right)$ determine a map $f_{k}: X-Z_{k} \rightarrow \mathbb{C P}^{2}$ with generic local models, canonically determined up to isotopy.

The model maps describing the local behavior of $f_{k}$ in approximately holomorphic local coordinates are now the following:

(0) $\left(z_{1}, \ldots, z_{n}\right) \mapsto\left(z_{1}: z_{2}: z_{3}\right)$ near a base point,

(i) $\left(z_{1}, \ldots, z_{n}\right) \mapsto\left(z_{1}, z_{2}\right)$,

(ii) $\quad\left(z_{1}, \ldots, z_{n}\right) \mapsto\left(z_{1}^{2}+\cdots+z_{n-1}^{2}, z_{n}\right)$,

(iii) $\left(z_{1}, \ldots, z_{n}\right) \mapsto\left(z_{1}^{3}-z_{1} z_{n}+z_{2}^{2}+\cdots+z_{n-1}^{2}, z_{n}\right)$. 
The set of critical points $R_{k} \subset X$ is again a (connected) smooth symplectic curve, and its image $D_{k}=f_{k}\left(R_{k}\right) \subset \mathbb{C P}^{2}$ is again a singular symplectic curve whose only singularities generically are transverse double points of either orientation and complex cusps. The fibers of $f_{k}$ are codimension 4 symplectic submanifolds, intersecting along $Z_{k}$; the fiber above a point of $\mathbb{C P}^{2}-D_{k}$ is smooth, while the fiber above a smooth point of $D_{k}$ presents an ordinary double point, the fiber above a node presents two ordinary double points, and the fiber above a cusp presents an $\mathrm{A}_{2}$ singularity.

The proof of these two results relies on a careful examination of the various possible local behaviors for the map $f_{k}$ and on transversality arguments establishing the existence of sections of $L^{\otimes k}$ with generic behavior. Hence, the argument relies on the enumeration of the various special cases, generic or not, that may occur; each one corresponds to the vanishing of a certain quantity that can be expressed in terms of the sections $s_{k}^{0}, s_{k}^{1}, s_{k}^{2}$ and their derivatives. Therefore, the proof largely reduces to a core ingredient which imitates classical singularity theory and can be thought of as a uniform transversality result for jets of approximately holomorphic sections $\mathrm{Au} 4$.

Given approximately holomorphic sections $s_{k}$ of very positive bundles $E_{k}$ (e.g. $\left.E_{k}=\mathbb{C}^{m} \otimes L^{\otimes k}\right)$ over the symplectic manifold $X$, one can consider the $r$-jets $j^{r} s_{k}=$ $\left(s_{k}, \partial s_{k},\left(\partial \partial s_{k}\right)_{\mathrm{sym}}, \ldots,\left(\partial^{r} s_{k}\right)_{\mathrm{sym}}\right)$, which are sections of the jet bundles $\mathcal{J}^{r} E_{k}=$ $\bigoplus_{j=0}^{r}\left(T^{*} X^{(1,0)}\right)_{\mathrm{sym}}^{\otimes j} \otimes E_{k}$. Jet bundles can naturally be stratified by approximately holomorphic submanifolds corresponding to the various possible local behaviors at order $r$ for the sections $s_{k}$. The generically expected behavior corresponds to the case where the jet $j^{r} s_{k}$ is transerse to the submanifolds in the stratification. The result is the following $\mathrm{Au} 4$ :

Theorem 3.5. Given stratifications $\mathcal{S}_{k}$ of the jet bundles $\mathcal{J}^{r} E_{k}$ by a finite number of approximately holomorphic submanifolds (Whitney-regular, uniformly transverse to fibers, and with curvature bounded independently of $k$ ), for large enough $k$ the vector bundles $E_{k}$ admit approximately holomorphic sections $s_{k}$ whose $r$-jets are uniformly transverse to the stratifications $\mathcal{S}_{k}$. Moreover these sections may be chosen arbitrarily close to given sections.

A one-parameter version of this result also holds, which makes it possible to obtain results of asymptotic uniqueness up to isotopy for generic sections.

Applied to suitably chosen stratifications, this result provides the main ingredient for the construction of $m$-tuples of approximately holomorphic sections of $L^{\otimes k}$ (and hence projective maps $f_{k}$ to $\mathbb{C P}^{m-1}$ ) with generic behavior. Once uniform transversality of jets has been obtained, the only remaining task is to achieve some control over the antiholomorphic derivative $\bar{\partial} f_{k}$ near the critical points of $f_{k}$ (typically its vanishing in some directions), in order to ensure that $\bar{\partial} f_{k} \ll \partial f_{k}$ everywhere; for low values of $m$ such as those considered above, this task is comparatively easy.

3.2. Monodromy invariants for branched covers of $\mathbb{C P}^{2}$. The topological data characterizing a symplectic branched covering $f: X^{4} \rightarrow \mathbb{C P}^{2}$ are on one hand the topology of the branch curve $D \subset \mathbb{C P}^{2}$ (up to isotopy and cancellation of pairs of nodes), and on the other hand a monodromy morphism $\theta: \pi_{1}\left(\mathbb{C P}^{2}-D\right) \rightarrow S_{N}$ describing the manner in which the $N=\operatorname{deg} f$ sheets of the covering are arranged above $\mathbb{C P}^{2}-D$. 
Some simple properties of the monodromy morphism $\theta$ can be readily seen by considering the local models of Definition 3.1 For example, the image of a small loop $\gamma$ bounding a disc that intersects $D$ transversely in a single smooth point (such a loop is called a geometric generator of $\pi_{1}\left(\mathbb{C P}^{2}-D\right)$ ) by $\theta$ is necessarily a transposition. The smoothness of $X$ above a singular point of $D$ implies some compatibility properties on these transpositions (geometric generators corresponding to the two branches of $D$ at a node must map to disjoint commuting transpositions, while to a cusp must correspond a pair of adjacent transpositions). Finally, the connectedness of $X$ implies the surjectivity of $\theta$ (because the $\operatorname{subgroup} \operatorname{Im}(\theta)$ is generated by transpositions and acts transitively on the fiber of the covering).

It must be mentioned that the amount of information present in the monodromy morphism $\theta$ is fairly small: a classical conjecture in algebraic geometry (Chisini's conjecture, essentially solved by Kulikov $[\mathrm{Ku}$ ) asserts that, given an algebraic singular plane curve $D$ with cusps and nodes, a symmetric group-valued monodromy morphism $\theta$ compatible with $D$ (in the above sense), if it exists, is unique except for a small list of low-degree counter-examples. Whether Chisini's conjecture also holds for symplectic branch curves is an open question, but in any case the number of possibilities for $\theta$ is always finite.

The study of a singular complex curve $D \subset \mathbb{C P}^{2}$ can be carried out using the braid monodromy techniques developed in complex algebraic geometry by Moishezon and Teicher [Mo2, Te1, .... : the idea is to choose a linear projection $\pi: \mathbb{C P}^{2}-\{\mathrm{pt}\} \rightarrow \mathbb{C P}^{1}$, for example $\pi(x: y: z)=(x: y)$, in such a way that the curve $D$ lies in general position with respect to the fibers of $\pi$, i.e. $D$ is positively transverse to the fibers of $\pi$ everywhere except at isolated non-degenerate smooth complex tangencies. The restriction $\pi_{\mid D}$ is then a singular branched covering of degree $d=\operatorname{deg} D$, with special points corresponding to the singularities of $D$ (nodes and cusps) and to the tangency points. Moreover, we can assume that all special points lie in distinct fibers of $\pi$. A plane curve satisfying these topological requirements is called a braided (or Hurwitz) curve.

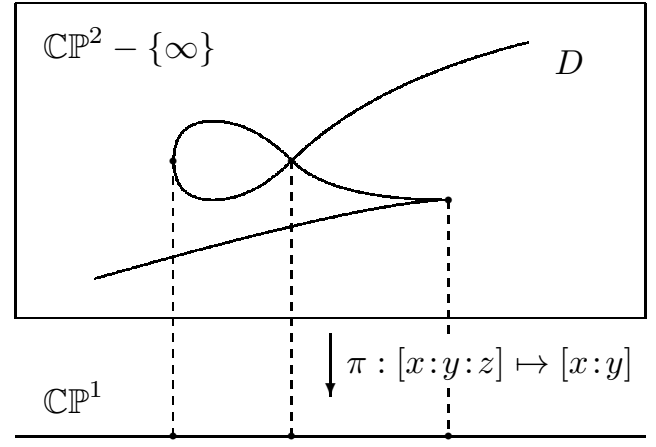

Except for those which contain special points of $D$, the fibers of $\pi$ are lines intersecting the curve $D$ in $d$ distinct points. If one chooses a reference point $q_{0} \in \mathbb{C P}^{1}$ (and the corresponding fiber $\ell \simeq \mathbb{C} \subset \mathbb{C P}^{2}$ of $\pi$ ), and if one restricts to an affine subset in order to be able to trivialize the fibration $\pi$, the topology of the branched covering $\pi_{\mid D}$ can be described by a braid monodromy morphism

$$
\rho: \pi_{1}\left(\mathbb{C}-\{\text { pts }\}, q_{0}\right) \rightarrow B_{d},
$$


where $B_{d}$ is the braid group on $d$ strings. The braid $\rho(\gamma)$ corresponds to the motion of the $d$ points of $\ell \cap D$ inside the fibers of $\pi$ when moving along the loop $\gamma$.

Recall that the braid group $B_{d}$ is the fundamental group of the configuration space of $d$ distinct points in $\mathbb{R}^{2}$; it is also the group of isotopy classes of compactly supported orientation-preserving diffeomorphisms of $\mathbb{R}^{2}$ leaving invariant a set of $d$ given distinct points. It is generated by the standard half-twists $X_{1}, \ldots, X_{d-1}$ (braids which exchange two consecutive points by rotating them counterclockwise by 180 degrees around each other), with relations $X_{i} X_{j}=X_{j} X_{i}$ for $|i-j| \geq 2$ and $X_{i} X_{i+1} X_{i}=X_{i+1} X_{i} X_{i+1}$ (the reader is referred to Birman's book Bir for more details).

Another equivalent way to consider the monodromy of a braided curve is to choose an ordered system of generating loops in the free group $\pi_{1}\left(\mathbb{C}-\{\right.$ pts $\left.\}, q_{0}\right)$. The morphism $\rho$ can then be described by a factorization in the braid group $B_{d}$, i.e. a decomposition of the monodromy at infinity into the product of the individual monodromies around the various special points of $D$. By observing that the total space of $\pi$ is the line bundle $O(1)$ over $\mathbb{C P}^{1}$, it is easy to see that the monodromy at infinity is given by the central element $\Delta^{2}=\left(X_{1} \ldots X_{d-1}\right)^{d}$ of $B_{d}$ (called "full twist" because it represents a rotation of a large disc by 360 degrees). The individual monodromies around the special points are conjugated to powers of half-twists, the exponent being 1 in the case of tangency points, 2 in the case of positive nodes (or -2 for negative nodes), and 3 in the case of cusps.

The braid monodromy $\rho$ and the corresponding factorization depend on trivialization choices, which affect them by simultaneous conjugation by an element of $B_{d}$ (change of trivialization of the fiber $\ell$ of $\pi$ ), or by Hurwitz operations (change of generators of the group $\left.\pi_{1}\left(\mathbb{C}-\{\mathrm{pts}\}, q_{0}\right)\right)$. There is a one-to-one correspondence between braid monodromy morphisms $\rho: \pi_{1}(\mathbb{C}-\{\mathrm{pts}\}) \rightarrow B_{d}$ (mapping generators to suitable powers of half-twists) up to these two algebraic operations and singular (not necessarily complex) braided curves of degree $d$ in $\mathbb{C P}^{2}$ up to isotopy among such curves (see e.g. KK for a detailed exposition). Moreover, it is easy to check that every braided curve in $\mathbb{C P}^{2}$ can be deformed into a braided symplectic curve, canonically up to isotopy among symplectic braided curves (this deformation is performed by collapsing the curve $D$ into a neighborhood of a complex line in a way that preserves the fibers of $\pi$ ). However, the curve $D$ is isotopic to a complex curve only for certain specific choices of the morphism $\rho$.

Unlike the case of complex curves, it is not clear a priori that the symplectic branch curve $D_{k}$ of one of the covering maps given by Theorem 3.3 can be made compatible with the linear projection $\pi$; making the curve $D_{k}$ braided relies on an improvement of the result in order to control more precisely the behavior of $D_{k}$ near its special points (tangencies, nodes, cusps). Moreover, one must take into account the possible occurrence of creations or cancellations of admissible pairs of nodes in the branch curve $D_{k}$, which affect the braid monodromy morphism $\rho_{k}: \pi_{1}(\mathbb{C}-\{\mathrm{pts}\}) \rightarrow B_{d}$ by insertion or deletion of pairs of factors. The uniqueness statement in Theorem 3.3 then leads to the following result [AK]:

Theorem 3.6 (A.-Katzarkov). For given large enough $k$, the monodromy morphisms $\left(\rho_{k}, \theta_{k}\right)$ associated to the approximately holomorphic branched covering maps $f_{k}: X \rightarrow \mathbb{C P}^{2}$ defined by triples of sections of $L^{\otimes k}$ are, up to conjugation, Hurwitz operations, and insertions/deletions, invariants of the symplectic manifold $(X, \omega)$. 
Moreover, these invariants are complete, in the sense that the data $\left(\rho_{k}, \theta_{k}\right)$ are sufficient to reconstruct the manifold $(X, \omega)$ up to symplectomorphism.

It is interesting to mention that the symplectic Lefschetz pencils constructed by Donaldson (Theorem 2.9) can be recovered very easily from the branched covering maps $f_{k}$, simply by considering the $\mathbb{C P}^{1}$-valued maps $\pi \circ f_{k}$. In other words, the fibers $\Sigma_{k, \alpha}$ of the pencil are the preimages by $f_{k}$ of the fibers of $\pi$, and the singular fibers of the pencil correspond to the fibers of $\pi$ through the tangency points of $D_{k}$.

In fact, the monodromy morphisms $\psi_{k}$ of the Lefschetz pencils $\pi \circ f_{k}$ can be recovered very explicitly from $\theta_{k}$ and $\rho_{k}$. By restriction to the line $\bar{\ell}=\ell \cup\{\infty\}$, the $S_{N}$-valued morphism $\theta_{k}$ describes the topology of a fiber $\Sigma_{k}$ of the pencil as an $N$-fold covering of $\mathbb{C P}^{1}$ with $d$ branch points; the set of base points $Z_{k}$ is the preimage of the point at infinity in $\bar{\ell}$. This makes it possible to define a lifting homomorphism from a subgroup $B_{d}^{0}\left(\theta_{k}\right) \subset B_{d}$ (liftable braids) to the mapping class group $\operatorname{Map}\left(\Sigma_{k}, Z_{k}\right)=\operatorname{Map}_{g, N}$. The various monodromies are then related by the following formula $\mathrm{AK}$ :

$$
\psi_{k}=\left(\theta_{k}\right)_{*} \circ \rho_{k} .
$$

The lifting homomorphism $\left(\theta_{k}\right)_{*}$ maps liftable half-twists to Dehn twists, so that the tangencies between the branch curve $D_{k}$ and the fibers of $\pi$ determine explicitly the vanishing cycles of the Lefschetz pencil $\pi \circ f_{k}$. On the other hand, the monodromy around a node or cusp of $D_{k}$ lies in the kernel of $\left(\theta_{k}\right)_{*}$.

The lifting homomorphism $\theta_{*}$ can be defined more precisely as follows: the space $\tilde{\mathcal{X}}_{d}$ of configurations of $d$ distinct points in $\mathbb{R}^{2}$ together with branching data (a transposition in $S_{N}$ attached to each point, or more accurately an $S_{N \text {-valued group }}$ homomorphism) is a finite covering of the space $\mathcal{X}_{d}$ of configurations of $d$ distinct points. The morphism $\theta$ determines a lift $\tilde{*}$ of the base point in $\mathcal{X}_{d}$, and the liftable braid subgroup of $B_{d}=\pi_{1}\left(\mathcal{X}_{d}, *\right)$ is the stabilizer of $\theta$ for the action of $B_{d}$ by deck transformations of the covering $\tilde{\mathcal{X}}_{d} \rightarrow \mathcal{X}_{d}$, i.e. $B_{d}^{0}(\theta)=\pi_{1}\left(\tilde{\mathcal{X}}_{d}, \tilde{*}\right)$. Moreover, $\tilde{\mathcal{X}}_{d}$ is naturally equipped with a universal fibration $\mathcal{Y}_{d} \rightarrow \tilde{\mathcal{X}}_{d}$ by genus $g$ Riemann surfaces with $N$ marked points: the lifting homomorphism $\theta_{*}: B_{d}^{0}(\theta) \rightarrow \operatorname{Map}_{g, N}$ is by definition the monodromy of this fibration.

The relation (3) is very useful for explicit calculations of the monodromy of Lefschetz pencils, which is accessible to direct methods only in a few very specific cases. By comparison, the various available techniques for braid monodromy calculations Mo3, Te1, ADKY are much more powerful, and make it possible to carry out calculations in a much larger number of cases (see below). In particular, in view of Donaldson's result we are mostly interested in the monodromy of high degree Lefschetz pencils, where the fiber genus and the number of singular fibers are very high, making them inaccessible to direct calculation even for the simplest complex algebraic surfaces.

When considering higher-dimensional manifolds, one may also associate monodromy invariants to a fibration $f_{k}: X \rightarrow \mathbb{C P}^{2}$; these consist of the braid monodromy of the critical curve $D_{k} \subset \mathbb{C P}^{2}$, and the monodromy of the fibration (with values in the mapping class group of the fiber). Furthermore, considering successive hyperplane sections and projections to $\mathbb{C P}^{2}$, one obtains a complete description of a symplectic manifold $\left(X^{2 n}, \omega\right)$ in terms of $n-1$ braid group-valued monodromy morphisms (describing the critical curves of various $\mathbb{C P}^{2}$-valued projections) and a single symmetric group-valued homomorphism (see Au3 for details). 
In principle, the above results reduce the classification of compact symplectic manifolds to purely combinatorial questions concerning braid groups and symmetric groups, and symplectic topology seems to largely reduce to the study of certain singular plane curves, or equivalently factorizations in braid groups.

The explicit calculation of these monodromy invariants is hard in the general case, but is made possible for a large number of complex surfaces by the use of "degeneration" techniques and of approximately holomorphic perturbations. These techniques make it possible to compute the braid monodromies of a variety of algebraic branch curves. In most cases, the calculation is only possible for a fixed projection to $\mathbb{C P}^{2}$ of a given algebraic surface (i.e., fixing $[\omega]$ and considering only $k=1$ ); see e.g. [Mo2, Ro, AGTV] …] for various such examples. In a smaller set of examples, the technique applies to projections of arbitrarily large degrees and hence makes it possible to compute explicitly the invariants defined by Theorem 3.6 To this date, the list consists of $\mathbb{C P}^{2}$ Mo5, Te3, $\mathbb{C P}^{1} \times \mathbb{C P}^{1}$ Mo3], certain Del Pezzo and K3 surfaces [Ro, CMT, the Hirzebruch surface $\mathbb{F}_{1}$ [MRT, ADKY], and all double covers of $\mathbb{C P}^{1} \times \mathbb{C P}^{1}$ branched along connected smooth algebraic curves, which includes an infinite family of surfaces of general type [ADKY].

The degeneration technique, developed by Moishezon and Teicher [Mo3, Te1, ....], starts with a projective embedding of the complex surface $X$, and deforms the image of this embedding to a singular configuration $X_{0}$ consisting of a union of planes intersecting along lines. The discriminant curve of a projection of $X_{0}$ to $\mathbb{C P}^{2}$ is therefore a union of lines; the manner in which the smoothing of $X_{0}$ affects this curve can be studied explicitly, by considering a certain number of standard local models near the various points of $X_{0}$ where three or more planes intersect (see [Te1 and ADKY] for detailed reviews of the technique). This method makes it possible to handle many examples in low degree, but in the case $k \gg 0$ that we are interested in (very positive linear systems over a fixed manifold), the calculations can only be carried out explicitly for very simple surfaces.

In order to proceed beyond this point, it becomes more efficient to move outside of the algebraic framework and to consider generic approximately holomorphic perturbations of non-generic algebraic maps; the greater flexibility of this setup makes it possible to choose more easily computable local models. For example, the direct calculation of the monodromy invariants becomes possible for all linear systems of the type $\pi^{*} O(p, q)$ on double couvers of $\mathbb{C P}^{1} \times \mathbb{C P}^{1}$ branched along connected smooth algebraic curves of arbitrary degree $\mathrm{ADKY}$. It also becomes possible to obtain a general "degree doubling" formula, describing explicitly the monodromy invariants associated to the linear system $L^{\otimes 2 k}$ in terms of those associated to the linear system $L^{\otimes k}$ (when $k \gg 0$ ), both for branched covering maps to $\mathbb{C P}^{2}$ and for 4-dimensional Lefschetz pencils AK2.

However, in spite of these successes, a serious obstacle restricts the practical applications of monodromy invariants: in general, they cannot be used efficiently to distinguish homeomorphic symplectic manifolds, because no algorithm exists to decide whether two words in a braid group or mapping class group are equivalent to each other via Hurwitz operations. Even if an algorithm could be found, another difficulty is due to the large amount of combinatorial data to be handled: on a typical interesting example, the braid monodromy data can already consist of $\sim 10^{4}$ factors in a braid group on $\sim 100$ strings for very small values of the parameter $k$, and the amount of data grows polynomially with $k$. 
Hence, even when monodromy invariants can be computed, they cannot be compared. This theoretical limitation makes it necessary to search for other ways to exploit monodromy data, e.g. by considering invariants that contain less information than braid monodromy but are easier to use in practice.

3.3. Fundamental groups of branch curve complements. Given a singular plane curve $D \subset \mathbb{C P}^{2}$, e.g. the branch curve of a covering, it is natural to study the fundamental group $\pi_{1}\left(\mathbb{C P}^{2}-D\right)$. The study of this group for various types of algebraic curves is a classical subject going back to the work of Zariski, and has undergone a lot of development in the 80's and 90's, in part thanks to the work of Moishezon and Teicher Mo2, Mo3, Te1, .... . The relation to braid monodromy invariants is a very direct one: the Zariski-van Kampen theorem provides an explicit presentation of the group $\pi_{1}\left(\mathbb{C P}^{2}-D\right)$ in terms of the braid monodromy morphism $\rho: \pi_{1}(\mathbb{C}-\{\mathrm{pts}\}) \rightarrow B_{d}$. However, if one is interested in the case of symplectic branch curves, it is important to observe that the introduction or the cancellation of pairs of nodes affects the fundamental group of the complement, so that it cannot be used directly to define an invariant associated to a symplectic branched covering. In the symplectic world, the fundamental group of the branch curve complement must be replaced by a suitable quotient, the stabilized fundamental group ADKY.

Using the same notations as above, the inclusion $i: \ell-\left(\ell \cap D_{k}\right) \rightarrow \mathbb{C P}^{2}-D_{k}$ of the reference fiber of the linear projection $\pi$ induces a surjective morphism on fundamental groups; the images of the standard generators of the free group $\pi_{1}\left(\ell-\left(\ell \cap D_{k}\right)\right)$ and their conjugates form a subset $\Gamma_{k} \subset \pi_{1}\left(\mathbb{C P}^{2}-D_{k}\right)$ whose elements are called geometric generators. Recall that the images of the geometric generators by the monodromy morphism $\theta_{k}$ are transpositions in $S_{N}$. The creation of a pair of nodes in the curve $D_{k}$ amounts to quotienting $\pi_{1}\left(\mathbb{C P}^{2}-D_{k}\right)$ by a relation of the form $\left[\gamma_{1}, \gamma_{2}\right] \sim 1$, where $\gamma_{1}, \gamma_{2} \in \Gamma_{k}$; however, this creation of nodes can be carried out by deforming the branched covering map $f_{k}$ only if the two transpositions $\theta_{k}\left(\gamma_{1}\right)$ and $\theta_{k}\left(\gamma_{2}\right)$ have disjoint supports. Let $K_{k}$ be the normal subgroup of $\pi_{1}\left(\mathbb{C P}^{2}-D_{k}\right)$ generated by all such commutators $\left[\gamma_{1}, \gamma_{2}\right]$. Then we have the following result $\mathrm{ADKY}$ :

Theorem 3.7 (A.-D.-K.-Y.). For given $k \gg 0$, the stabilized fundamental group $\bar{G}_{k}=\pi_{1}\left(\mathbb{C P}^{2}-D_{k}\right) / K_{k}$ is an invariant of the symplectic manifold $\left(X^{4}, \omega\right)$.

This invariant can be calculated explicitly for the various examples where monodromy invariants are computable $\left(\mathbb{C P}^{2}, \mathbb{C P}^{1} \times \mathbb{C P}^{1}\right.$, some Del Pezzo and K3 surfaces, Hirzebruch surface $\mathbb{F}_{1}$, double covers of $\left.\mathbb{C P}^{1} \times \mathbb{C P}^{1}\right)$; namely, the extremely complicated presentations given by the Zariski-van Kampen theorem in terms of braid monodromy data can be simplified in order to obtain a manageable description of the fundamental group of the branch curve complement. These examples lead to various observations and conjectures.

A first remark to be made is that, for all known examples, when the parameter $k$ is sufficiently large the stabilization operation becomes trivial, i.e. geometric generators associated to disjoint transpositions already commute in $\pi_{1}\left(\mathbb{C P}^{2}-D_{k}\right)$, so that $K_{k}=\{1\}$ and $\bar{G}_{k}=\pi_{1}\left(\mathbb{C P}^{2}-D_{k}\right)$. For example, in the case of $X=\mathbb{C P}^{2}$ with its standard Kähler form, we have $\bar{G}_{k}=\pi_{1}\left(\mathbb{C P}^{2}-D_{k}\right)$ for all $k \geq 3$. Therefore, when $k \gg 0$ no information seems to be lost when quotienting by $K_{k}$ (the situation for small values of $k$ is very different). 
The following general structure result can be proved for the groups $\bar{G}_{k}$ (and hence for $\left.\pi_{1}\left(\mathbb{C P}^{2}-D_{k}\right)\right)$ [ADKY]:

Theorem 3.8 (A.-D.-K.-Y.). Let $f:(X, \omega) \rightarrow \mathbb{C P}^{2}$ be a symplectic branched covering of degree $N$, with braided branch curve $D$ of degree $d$, and let $\bar{G}=\pi_{1}\left(\mathbb{C P}^{2}-\right.$ $D) / K$ be the stabilized fundamental group of the branch curve complement. Then there exists a natural exact sequence

$$
1 \longrightarrow G^{0} \longrightarrow \bar{G} \longrightarrow S_{N} \times \mathbb{Z}_{d} \longrightarrow \mathbb{Z}_{2} \longrightarrow 1 \text {. }
$$

Moreover, if $X$ is simply connected then there exists a natural surjective homomorphism $\phi: G^{0} \rightarrow\left(\mathbb{Z}^{2} / \Lambda\right)^{n-1}$, where

$$
\Lambda=\left\{\left(c_{1}\left(K_{X}\right) \cdot \alpha,\left[f^{-1}(\bar{\ell})\right] \cdot \alpha\right), \alpha \in H_{2}(X, \mathbb{Z})\right\} .
$$

In this statement, the two components of the morphism $\bar{G} \rightarrow S_{N} \times \mathbb{Z}_{d}$ are respectively the monodromy of the branched covering, $\theta: \pi_{1}\left(\mathbb{C P}^{2}-D\right) \rightarrow S_{N}$, and the linking number (or abelianization, when $D$ is irreducible) morphism

$$
\delta: \pi_{1}\left(\mathbb{C P}^{2}-D\right) \rightarrow \mathbb{Z}_{d}\left(\simeq H_{1}\left(\mathbb{C P}^{2}-D, \mathbb{Z}\right)\right) .
$$

The subgroup $\Lambda$ of $\mathbb{Z}^{2}$ is entirely determined by the numerical properties of the canonical class $c_{1}\left(K_{X}\right)$ and of the hyperplane class (the homology class of the preimage of a line $\bar{\ell} \subset \mathbb{C P}^{2}$ : in the case of the covering maps of Theorem 3.3 we have $\left.\left[f^{-1}(\bar{\ell})\right]=c_{1}\left(L^{\otimes k}\right)=\frac{k}{2 \pi}[\omega]\right)$. The morphism $\phi$ is defined by considering the $N$ lifts in $X$ of a closed loop $\gamma$ belonging to $G^{0}$, or more precisely their homology classes (whose sum is trivial) in the complement of a hyperplane section and of the ramification curve in $X$.

Moreover, in the known examples we have a much stronger result on the structure of the subgroups $G_{k}^{0}$ for the branch curves of large degree covering maps (determined by sufficiently ample linear systems) [ADKY].

Say that the simply connected complex surface $(X, \omega)$ belongs to the class $(\mathcal{C})$ if it belongs to the list of computable examples: $\mathbb{C P}^{1} \times \mathbb{C P}^{1}, \mathbb{C P}^{2}$, the Hirzebruch surface $\mathbb{F}_{1}$ (equipped with any Kähler form), a Del Pezzo or K3 surface (equipped with a Kähler form coming from a specific complete intersection realization), or a double cover of $\mathbb{C P}^{1} \times \mathbb{C P}^{1}$ branched along a connected smooth algebraic curve (equipped with a Kähler form in the class $\pi^{*} O(p, q)$ for $p, q \geq 1$ ). Then we have:

Theorem 3.9 (A.-D.-K.-Y.). If $(X, \omega)$ belongs to the class $(\mathcal{C})$, then for all large enough $k$ the homomorphism $\phi_{k}$ induces an isomorphism on the abelianized groups, i.e. $\mathrm{Ab} G_{k}^{0} \simeq\left(\mathbb{Z}^{2} / \Lambda_{k}\right)^{N_{k}-1}$, while $\operatorname{Ker} \phi_{k}=\left[G_{k}^{0}, G_{k}^{0}\right]$ is a quotient of $\mathbb{Z}_{2} \times \mathbb{Z}_{2}$.

It is natural to make the following conjecture:

Conjecture 3.10. If $X$ is a simply connected symplectic 4-manifold, then for all large enough $k$ the homomorphism $\phi_{k}$ induces an isomorphism on the abelianized groups, i.e. $\mathrm{Ab} G_{k}^{0} \simeq\left(\mathbb{Z}^{2} / \Lambda_{k}\right)^{N_{k}-1}$.

3.4. Symplectic isotopy and non-isotopy. While it has been well-known for many years that compact symplectic 4-manifolds do not always admit Kähler structures, it has been discovered more recently that symplectic curves (smooth or singular) in a given manifold can also offer a wider range of possibilities than complex curves. Proposition 3.2 and Theorem 3.3 establish a bridge between these two phenomena: indeed, a covering of $\mathbb{C P}^{2}$ (or any other complex surface) branched along a complex curve automatically inherits a complex structure. Therefore, starting with 
a non-Kähler symplectic manifold, Theorem 3.3 always yields branch curves that are not isotopic to any complex curve in $\mathbb{C P}^{2}$. The study of isotopy and non-isotopy phenomena for curves is therefore of major interest for our understanding of the topology of symplectic 4-manifolds.

The symplectic isotopy problem asks whether, in a given complex surface, every symplectic submanifold representing a given homology class is isotopic to a complex submanifold. The first positive result in this direction was due to Gromov, who showed using his compactness result for pseudo-holomorphic curves (Theorem 1.10) that, in $\mathbb{C P}^{2}$, a smooth symplectic curve of degree 1 or 2 is always isotopic to a complex curve. Successive improvements of this technique have made it possible to extend this result to curves of higher degree in $\mathbb{C P}^{2}$ or $\mathbb{C P}^{1} \times \mathbb{C P}^{1}$; the currently best known result is due to Siebert and Tian, and makes it possible to handle the case of smooth curves in $\mathbb{C P}^{2}$ up to degree 17 ST] (see also their lecture notes in this volume). Isotopy results are also known for sufficiently simple singular curves (Barraud, Shevchishin [Sh, ...).

Contrarily to the above examples, the general answer to the symplectic isotopy problem appears to be negative. The first counterexamples among smooth connected symplectic curves were found by Fintushel and Stern [FS], who constructed by a braiding process infinite families of mutually non-isotopic symplectic curves representing a same homology class (a multiple of the fiber) in elliptic surfaces (a similar construction can also be performed in higher genus). However, these two constructions are preceded by a result of Moishezon [Mo4, who established in the early 90's a result implying the existence in $\mathbb{C P}^{2}$ of infinite families of pairwise nonisotopic singular symplectic curves of given degree with given numbers of node and cusp singularities. A reformulation of Moishezon's construction makes it possible to see that it also relies on braiding; moreover, the braiding construction can be related to a surgery operation along a Lagrangian torus in a symplectic 4-manifold, known as Luttinger surgery ADK]. This reformulation makes it possible to vastly simplify Moishezon's argument, which was based on lengthy and delicate calculations of fundamental groups of curve complements, while relating it with various constructions developed in 4-dimensional topology.

Given an embedded Lagrangian torus $T$ in a symplectic 4-manifold $(X, \omega)$, a homotopically non-trivial embedded loop $\gamma \subset T$ and an integer $k$, Luttinger surgery is an operation that consists in cutting out from $X$ a tubular neighborhood of $T$, foliated by parallel Lagrangian tori, and gluing it back in such a way that the new meridian loop differs from the old one by $k$ twists along the loop $\gamma$ (while longitudes are not affected), yielding a new symplectic manifold $(\tilde{X}, \tilde{\omega})$. This relatively littleknown construction, which e.g. makes it possible to turn a product $T^{2} \times \Sigma$ into any surface bundle over $T^{2}$, or to transform an untwisted fiber sum into a twisted one, can be used to described in a unified manner numerous examples of exotic symplectic 4-manifolds constructed in the past few years.

Meanwhile, the braiding construction of symplectic curves starts with a (possibly singular) symplectic curve $\Sigma \subset\left(Y^{4}, \omega_{Y}\right)$ and two symplectic cylinders embedded in $\Sigma$, joined by a Lagrangian annulus contained in the complement of $\Sigma$, and consists in performing $k$ half-twists between these two cylinders in order to obtain a new symplectic curve $\tilde{\Sigma}$ in $Y$. 


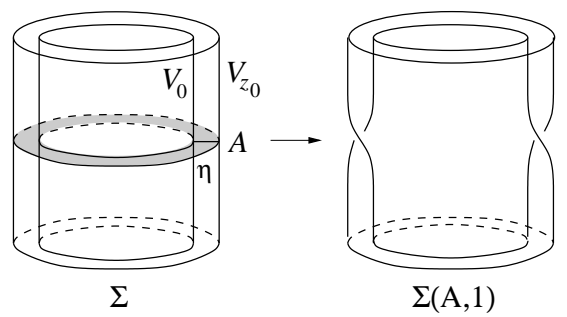

When $\Sigma$ is the branch curve of a symplectic branched covering $f: X \rightarrow Y$, the following result holds [ADK]:

Proposition 3.11. The covering of $Y$ branched along the symplectic curve $\tilde{\Sigma}$ obtained by braiding $\Sigma$ along a Lagrangian annulus $A \subset Y-\Sigma$ is naturally symplectomorphic to the manifold $\tilde{X}$ obtained from the branched cover $X$ by Luttinger surgery along a Lagrangian torus $T \subset X$ formed by the union of two lifts of $A$.

Hence, once an infinite family of symplectic curves has been constructed by braiding, it is sufficient to find invariants that distinguish the corresponding branched covers in order to conclude that the curves are not isotopic. In the Fintushel-Stern examples, the branched covers are distinguished by their Seiberg-Witten invariants, whose behavior is well understood in the case of elliptic fibrations and their surgeries.

In the case of Moishezon's examples, a braiding construction makes it possible to construct, starting from complex curves $\Sigma_{p, 0} \subset \mathbb{C P}^{2}(p \geq 2)$ of degree $d_{p}=9 p(p-1)$ with $\kappa_{p}=27(p-1)(4 p-5)$ cusps and $\nu_{p}=27(p-1)(p-2)\left(3 p^{2}+3 p-8\right) / 2$ nodes, symplectic curves $\Sigma_{p, k} \subset \mathbb{C P}^{2}$ for all $k \in \mathbb{Z}$, with the same degree and numbers of singular points. By Proposition 3.11 these curves can be viewed as the branch curves of symplectic coverings whose total spaces $X_{p, k}$ differ by Luttinger surgeries along a Lagrangian torus $T \subset X_{p, 0}$. The effect of these surgeries on the canonical class and on the symplectic form can be described explicitly, which makes it possible to distinguish the manifolds $X_{p, k}$ : the canonical class of $\left(X_{p, k}, \omega_{p, k}\right)$ is given by $p c_{1}\left(K_{p, k}\right)=(6 p-9)\left[\omega_{p, k}\right]+(2 p-3) k P D([T])$. Moreover, $[T] \in H_{2}\left(X_{p, k}, \mathbb{Z}\right)$ is not a torsion class, and if $p \not \equiv 0 \bmod 3$ or $k \equiv 0 \bmod 3$ then it is a primitive class ADK. This implies that infinitely many of the curves $\Sigma_{p, k}$ are pairwise non-isotopic.

It is to be observed that the argument used by Moishezon to distinguish the curves $\Sigma_{p, k}$, which relies on a calculation of the fundamental groups $\pi_{1}\left(\mathbb{C P}^{2}-\Sigma_{p, k}\right)$ Mo4, is related to the one in ADK by means of Conjecture 3.10 of which it can be concluded a posteriori that it is satisfied by the given branched covers $X_{p, k} \rightarrow \mathbb{C P}^{2}$ : in particular, the fact that $\pi_{1}\left(\mathbb{C P}^{2}-\Sigma_{p, k}\right)$ is infinite for $k=0$ and finite for $k \neq 0$ is consistent with the observation that the canonical class of $X_{p, k}$ is proportional to its symplectic class iff $k=0$.

\section{SympleCtiC SURFACES From Symmetric PROduCts}

This section will describe an approach, developed in [DS, Sm4 and [S. to various theorems due to Taubes, but in the context of Lefschetz pencils rather than Seiberg-Witten gauge theory and the equivalence "SW=Gr". The main result on which we will focus is Theorem 1.11 $(i)$ about the existence of embedded symplectic submanifolds representing the canonical class (although we will establish the result 
only in the case where $\left.b_{+}>1+b_{1}\right)$. Note that a Lefschetz pencil is a family of symplectic surfaces of arbitrarily large volume and complexity (depending on $k$ ), so somewhat different techniques are needed to find symplectic surfaces in a prescribed homology class.

4.1. Symmetric products. Let $\left(X, \omega_{X}\right)$ be a symplectic 4-manifold with integral $\left[\omega_{X}\right]$, and fix a Lefschetz pencil, giving rise to a Lefschetz fibration $f: \hat{X} \rightarrow S^{2}$ on the blow-up. We will always equip $\hat{X}$ with the symplectic form $\omega_{C}=p^{*} \omega_{X}+$ $C f^{*} \omega_{S^{2}}$ for some large $C>0$.

Definition 4.1. A standard surface in $\hat{X}$ is a smooth embedded surface $\Sigma$ such that $f_{\mid \Sigma}$ is a simple branched covering over $S^{2}$, and at each branch point df gives an oriented isomorphism between the normal bundle to $\Sigma$ and $T S^{2}$.

The condition on $d f$ ensures that we have positive branching, that is, near each branch point there are complex-valued coordinates such that $\Sigma$ looks like $\left\{\left(z_{1}, z_{2}\right) \in\right.$ $\left.\mathbb{C}^{2}, z_{1}^{2}=z_{2}\right\}$, with $f$ being the projection to $z_{2}$.

Lemma 4.2. A standard surface in $\hat{X}$, disjoint from the exceptional sections $E_{i}$, defines a symplectic surface in $X$.

This follows from a local calculation showing that $\left(\hat{X}-\bigcup E_{i}, \omega_{C}\right)$ is symplectomorphic to $\left(X-\left\{b_{i}\right\},(1+k C) \omega_{X}\right)$, where $k$ is the degree of the pencil. By taking $C$ sufficiently large it is clear that the given standard surface is symplectic in $\left(\hat{X}, \omega_{C}\right)$.

Rather than encoding a standard surface algebraically in the monodromy representation, we will take a geometric standpoint and obtain such surfaces from sections of a "relative symmetric product". This construction will in fact yield singular unions of such surfaces, so for later use we therefore provide a smoothing lemma. As a piece of notation, a positive symplectic divisor is a union of symplectic surfaces $D=\sum a_{i} \Sigma_{i}$ with $a_{i}>0$, with the $\Sigma_{i}$ pairwise having only isolated positive transverse intersections, and no triple intersections. (In fact, a careful inspection of the later argument will show that, in the positive divisors we construct, if $\Sigma_{i}$ and $\Sigma_{j}$ intersect, then either one is an exceptional sphere, or one has multiplicity $a_{i}>1$.) In any case, we will be able to use the following criterion:

Proposition 4.3. If a symplectic divisor satisfies $D \cdot \Sigma_{j} \geq 0$ for all $j$, then it can be symplectically smoothed.

Sketch of proof. Suppose first that all the $\Sigma_{i}$ are complex curves in a Kähler surface. The hypothesis says that the line bundle $O(D)$ has non-negative degree on each component. Then we can find a smooth section $\gamma$ of $O(D)$ which is holomorphic near each intersection point and near its (transverse) zero set. If the section $s$ defines the divisor $D$, then the zero set of $s-\epsilon \gamma$ is a suitable symplectic smoothing for sufficiently small $\epsilon$.

In general, the $\Sigma_{i}$ may not be symplectomorphic to any complex model. Near an intersection point of $\Sigma$ ad $\Sigma^{\prime}$, write $\Sigma^{\prime}$ as a graph of a linear map $A$ over the symplectic orthogonal of $\Sigma$. The symplecticity of $\Sigma^{\prime}$ implies that $\operatorname{det}(A)>-1$; for a complex model (in which $A$ is an element of $\mathbb{C} \subset \operatorname{End}\left(\mathbb{R}^{2}\right)$ ), we need $\operatorname{det}(A)>0$ (or $A=0$ ). This can be achieved by a small compactly supported perturbation of $\Sigma^{\prime}$. 
We now turn to the main construction. The $d$-fold symmetric prouct of a Riemann surface is canonically a smooth complex manifold of dimension $d$. A holomorphic atlas is provided by noticing that the elementary symmetric functions define a biholomorphism $\operatorname{Sym}^{d}(\mathbb{C})=\mathbb{C}^{d} / S_{d} \stackrel{\sim}{\longrightarrow} \mathbb{C}^{d}$. For instance, taking a non-zero $(d+1)$-tuple of numbers to the roots of the degree $d$ polynomial having these as its coefficients gives an identification between $\mathbb{C P}^{d}$ and $\operatorname{Sym}^{d}\left(\mathbb{C P}^{1}\right)$.

Note that the symmetric product of a smooth two-manifold has no natural smooth atlas, although it is well-defined up to non-canonical diffeomorphisms. We will always work with almost-complex structures on our Lefschetz fibrations which make the projection map pseudo-holomorphic (call these almost-complex structures fibered). In this case the fibers become Riemann surfaces.

Theorem 4.4. Given a Lefschetz fibration $f: \hat{X} \rightarrow \mathbb{C P}^{1}$ with a fibered almostcomplex structure, for each $d \geq 0$ there is a smooth compact symplectic manifold $X_{d}(f) \rightarrow \mathbb{C P}^{1}$ with fiber at $t \notin \operatorname{crit}(f)$ the symmetric product $\operatorname{Sym}^{d}\left(f^{-1}(t)\right)$.

Away from the singular fibers, this follows by taking charts on $X$ of the form $\theta: D_{1} \times D_{2} \rightarrow X$, such that: for each $x \in D_{1}, \theta_{x}:\{x\} \times D_{2} \rightarrow X$ gives a holomorphic disc in a fiber of $f$; and $f \circ \theta=i \circ \pi_{1}$, for $i$ an inclusion $D_{1} \hookrightarrow \mathbb{C P}^{1}$. In other words, the fibers of $f$ are Riemann surfaces whose complex structures vary smoothly in the obvious way. Now we restrict further to work with almost complex structures which are integrable near the singular fibers. In this case, we can fill in the fibration by appealing to algebraic geometry. Namely, replace the symmetric product (a moduli space of structure sheaves) by a Hilbert scheme (a moduli space of ideal sheaves). We will use a concrete description of the Hilbert scheme of $\mathbb{C}^{2}$ together with our local model for the singularities of $f$.

Definition 4.5. Hilb ${ }_{d}\left(\mathbb{C}^{2}\right)$ comprises the triples $\left\{B_{1}, B_{2}, g\right\} / \sim$ where $B_{1}, B_{2}$ are $d \times d$ commuting matrices, and $g$ is a vector which is not contained in any subspace $S \subset \mathbb{C}^{d}$ stabilized by both $B_{i} . G L_{d}(\mathbb{C})$ acts by conjugating the $B_{i}$ 's and on the left of $g$.

To unravel this, think of an ideal $J \subset \mathbb{C}\left[z_{1}, z_{2}\right]$ of codimension $d$. Identify $\mathbb{C}\left[z_{1}, z_{2}\right] / J=\mathbb{C}^{d}$, and define $B_{1}, B_{2}$ to be the action of $z_{1}, z_{2}$, and $g$ the image of 1 . Conversely, given a triple, define a map $\mathbb{C}\left[z_{1}, z_{2}\right] \rightarrow \mathbb{C}^{d}$ by $f\left(z_{1}, z_{2}\right) \mapsto f\left(B_{1}, B_{2}\right) g$. The stability condition on $g$ implies that this is surjective, and the kernel gives an ideal of codimension $d$. Nakajima's notes $\mathrm{Na}$ show that this correspondence is indeed a holomorphic parametrisation of the Hilbert scheme of length $d$ subschemes of $\mathbb{C}^{2}$ by triples of matrices.

Now consider the relative version of this, for the map $\mathbb{C}^{2} \rightarrow \mathbb{C},\left(z_{1}, z_{2}\right) \mapsto z_{1} z_{2}$. The fiber over $t$ is by definition those ideals which contain $z_{1} z_{2}-t$, giving

$$
B_{1} B_{2}=t I, \quad B_{2} B_{1}=t I, \quad \text { plus stability. }
$$

If the $B_{i}$ are simultaneously diagonalizable, we get a $d$-tuple of pairs of eigenvalues, i.e. a point of $S y m^{d}\left(f^{-1}(t)\right)$. More generally, the $B_{i}$ can be represented as a pair of upper triangular matrices, and again there is a map to the symmetric product given by taking eigenvalues. For $t \neq 0$ this map is an isomorphism because of the stability condition; for $t=0$ this is no longer the case, and suitable off-diagonal matrix entries become coordinates on the fibers. In any case, we now have explicit equations defining $X_{d}(f)$; one can use these to give a concrete description of its topology, and show smoothness. 
Proposition 4.6. The total space of this relative Hilbert scheme is smooth.

Sketch of proof. One can show (for instance from the Abel-Jacobi discussion below) that the $t=0$ fiber has normal crossings. Given this, at each point of the singular locus there are two line bundles $\nu_{i}$ - the normal bundles to the two branches whose intersection defines the singular locus - and the given deformation of the singular fiber gives a section of $\nu_{1}^{*} \otimes \nu_{2}^{*}$. The total space is smooth if this section has no zeros. In our case, the $\nu_{i}$ are canonically identified with the tangent spaces at the node of the singular fiber of $f$, so the bundle $\nu_{1}^{*} \otimes \nu_{2}^{*}$ is trivial. Hence we only need check smoothness at one point. This one can do by writing down explicit local sections of the map.

At least when we assume that all the singular fibers of $f$ are irreducible, we get another point of view from the Abel-Jacobi map. Let $\Sigma$ be a Riemann surface.

Definition 4.7. The Abel-Jacobi map $\operatorname{Sym}^{d} \Sigma \rightarrow \operatorname{Pic}^{d}(\Sigma)$ takes a divisor $D$ to the line bundle $\mathcal{O}(D)$.

The key point is to identify the fibers of this map with linear systems $\mathbb{P}\left(H^{0}(L)\right)$, hence projective spaces whose dimension may vary as $L$ moves in Pic.

Example. Let $d=2 g-2$ (by the adjunction formula, this is the relevant case if a standard surface in $\hat{X}$ is to represent $K_{\hat{X}}$ ). From the Riemann-Roch theorem $h^{0}(L)-h^{1}(L)=d-g+1=g-1$, whilst by Serre duality, $h^{1}(L)>0$ only if $L=K$ (since only the trivial degree 0 bundle has a section). Thus $\operatorname{Sym}^{2 g-2}(\Sigma) \rightarrow$ $\operatorname{Pic}^{2 g-2}(\Sigma)$ is a (locally trivial) $\mathbb{C P}^{g-2}$-bundle away from a single exceptional fiber, which is a $\mathbb{C P}^{g-1}$. For instance, $\operatorname{Sym}^{2}\left(\Sigma_{2}\right) \rightarrow T^{4}$ blows down a single exceptional sphere.

A line bundle on a nodal Riemann surface $\Sigma_{0}$ is given by a line bundle on its normalization $\tilde{\Sigma}_{0}$ together with a $\mathbb{C}^{*}$ gluing parameter $\lambda$ to identify the fibers over the preimages of the node. This $\mathbb{C}^{*}$-bundle over $T^{2 g-2}$ naturally compactifies to a $\mathbb{C P}^{1}$-bundle; if we glue the 0 and $\infty$ sections of this over a translation in the base, we compactify the family of Picard varieties for a family of curves with an irreducible nodal fiber. (Think of an elliptic Lefschetz fibration with section, which does precisely this in the $g=1$ case!) Thus, under our assumptions, the relative Picard fibration $P_{d}(f)$ is also a smooth compact symplectic manifold.

Given $L \in \operatorname{Pic}^{2 g-2}\left(\Sigma_{0}\right)$ in the smooth locus, the line bundle on the normalization has a space of sections of dimension $g$. Hence the $\lambda$-hyperplane of sections which transform correctly over the node gives a $\mathbb{C P}^{g-2}$. The situation is similar along the normal crossing divisor in Pic (just take $\lambda=0$ or $\infty$ ), but changes if $L$ gives rise to the canonical bundle of the normalization, where the dimension jumps. Summing up:

Proposition 4.8. The symplectic manifold $X_{2 g-2}(f)$ is the total space of a family of projective spaces over $P_{2 g-2}(f)$, the family being a locally trivial $\mathbb{C P}^{g-1}$ bundle over the section defined by the canonical line bundles of the fibers of $f$ and a locally trivial $\mathbb{C P}^{g-2}$ bundle away from this section.

There is an analogous description for other values of $d$, and one sees that the singular locus of the singular fiber of $X_{d}(f)$ is a copy of $\operatorname{Sym}^{d-1}\left(\tilde{\Sigma}_{0}\right)$. It may be helpful to point out that tuples set-theoretically including the node are not the same thing as singular points of $\operatorname{Hilb}\left(\Sigma_{0}\right)$. The relevance is that a smooth family 
of surfaces in $X$ may well include members passing through critical points of $f$, but smooth sections of $X_{d}(f)$ can never pass through the singular loci of fibers. (Roughly speaking, the singular locus of $\mathrm{Hilb}_{d}\left(\Sigma_{0}\right)$ comprises tuples which contain the node with odd multiplicity.)

To see that $X_{d}(f)$ admits symplectic structures, one uses an analogue of the argument that applies to Lefschetz fibrations - we have a family of Kähler manifolds with locally holomorphic singularities, so we can patch together local forms to obtain something vertically non-degenerate and then add the pull-back of a form from the base.

4.2. Taubes' theorem. The construction of symplectic surfaces representing $\left[K_{X}\right]$ proceeds in two stages. Let $r=2 g-2$. A section $s$ of $X_{r}(f)$ defines a cycle $C_{s} \subset \hat{X}$. There is a homotopy class $h$ of sections such that $\left[C_{h}\right]=P D\left(-c_{1}(\hat{X})\right)$. First, we show that the Gromov invariant counting sections of $X_{r}(f)$ in the class $h$ is nonzero, and then from the pseudoholomorphic sections of $X_{r}(f)$ thereby provided we construct standard surfaces (which have a suitable component which descends from $\hat{X}$ to $X)$. For the first stage the Abel-Jacobi map is the key, whilst for the second we work with almost complex structures compatible with the "diagonal" strata of the symmetric products (tuples of points not all of which are pairwise distinct).

That the Gromov invariant is well-defined follows easily from a description of the possible bubbles arising from cusp curves in $X_{r}(f)$. Again, we will always use "fibered" almost complex structures $J$ on $X_{r}(f)$, i.e. ones making the projection to $\mathbb{C P}^{1}$ holomorphic.

Lemma 4.9. $\pi_{2}\left(\operatorname{Sym}^{r}(\Sigma)\right)=\mathbb{Z}$, generated by a line $l$ in a fiber of the Abel-Jacobi map. All bubbles in cusp limits of sections of $X_{r}(f) \rightarrow \mathbb{C P}^{1}$ are homologous to a multiple of $l$.

Sketch of proof. The first statement follows from the Lefschetz hyperplane theorem and the fact that $\operatorname{Sym}^{d}(\Sigma)$ is a projective bundle for $d>r$; it is also an easy consequence of the Dold-Thom theorem in algebraic topology. The second statement is then obvious away from singular fibers, but these require special treatment, cf. DS.

Since $\left\langle c_{1}\left(\operatorname{Sym}^{r}(\Sigma)\right), h\right\rangle>0$ this shows that spaces of holomorphic sections can be compactified by high codimension pieces, after which it is easy to define the Gromov invariant.

Proposition 4.10. For a suitable $J$, the moduli space of $J$-holomorphic sections in the class $h$ is a projective space of dimension $\left(b_{+}-b_{1}-1\right) / 2-1$.

Proof. The index of the $\bar{\partial}$-operator on $f_{*} K \rightarrow \mathbb{C P}^{1}$ is $\left(b_{+}-b_{1}-1\right) / 2$. Choose $J$ on $X_{r}(f)$ so that the projection $\tau$ to $P_{r}(f)$ is holomorphic, and the latter has the canonical section $s_{K}$ as an isolated holomorphic section (the index of the normal bundle to this is $\left.-\left(b_{+}-b_{1}-1\right) / 2\right)$. Now all holomorphic sections of $X_{r}(f)$ lie over $s_{K}$. If the degree of the original Lefschetz pencil was sufficiently large, the rank of the bundle $f_{*} K$ is much larger than its first Chern class. Then, for a generic connection, the bundle becomes $\mathcal{O} \oplus \mathcal{O} \oplus \cdots \oplus \mathcal{O}(-1) \oplus \cdots \oplus \mathcal{O}(-1)$ (by Grothendieck's theorem). Hence, all nonzero sections are nowhere zero, so yield homotopic sections of the projectivisation $\mathbb{P}\left(f_{*} K\right)=\tau^{-1}\left(s_{K}\right)$, each defining a cycle in $\hat{X}$ in the fixed homology class $-c_{1}(\hat{X})$. 
We now have a $J$ such that $\mathcal{M}_{J}(h)$ is compact and smooth - but of the wrong dimension (index arguments show that for a section $s$ of $X_{r}(f)$ giving a cycle $C_{s}$ in $X$, the space of holomorphic sections has virtual dimension $C_{s} \cdot C_{s}-C_{s} \cdot K_{X}$ ). The actual invariant is given as the Euler class of an obstruction bundle, whose fiber at a curve $C$ is $H^{1}\left(\nu_{C}\right)$ (from the above, all our curves are embedded so this bundle is well-defined). Let $Q$ be the quotient bundle over $\mathbb{C P}^{n}$ (defined as the cokernel of the inclusion of the tautological bundle into $\left.\underline{\mathbb{C}}^{n+1}\right)$. It has Euler class $(-1)^{n+1}$.

Lemma 4.11. For $J$ as above, the obstruction bundle Obs $\rightarrow \mathcal{M}_{J}(h)$ is $Q \rightarrow \mathbb{C P}^{N}$ with $N=\left(b_{+}-b_{1}-1\right) / 2-1$.

Sketch of proof. It is not hard to show that there is a global holomorphic model $M\left(f_{*} K\right)$ for the map $\tau: X_{r}(f) \rightarrow P_{r}(f)$ in a neighborhood of the fibers of excess dimension, i.e. $\tau^{-1}\left(s_{K}\right)=\mathbb{P}\left(f_{*} K\right)$. In other words, the obstruction computation reduces to algebraic geometry (hence our notation for the bundle $f_{*} K \rightarrow \mathbb{C P}^{1}$ with fiber $\left.H^{0}\left(K_{\Sigma}\right)\right)$. We have a sequence of holomorphic vector bundles

$$
0 \rightarrow T\left(\mathbb{P}\left(f_{*} K\right)\right) \rightarrow T M\left(f_{*} K\right) \rightarrow T(W) \rightarrow \operatorname{coker}(D \tau) \rightarrow 0
$$

where $W$ is fiberwise dual to $f_{*} K$, but globally twisted by $\mathcal{O}(-2)$ (this is because $K_{\hat{X}} \mid f^{-1}(t)$ is not canonically $K_{f^{-1}(t)}$, introducing a twist into all the identifications). If $\tau$ was actually a submersion, clearly the obstruction bundle would be trivial (as $\nu_{C}$ would be pulled back from $\tau(C)=s_{K} \subset P_{r}(f)$ ); the deviation from this is measured by $H^{1}(\operatorname{coker}(D \tau))$. We compute this by splitting the above sequence into two short exact sequences and take the long exact sequences in cohomology.

Summing up, when $b_{+}>1+b_{1}(X)$, we have shown that the Gromov invariant for the class $h$ is \pm 1 . (One can improve the constraint to $b_{+}>2$, but curiously it seems tricky to exactly reproduce Taubes' sharp bound $b_{+} \geq 2$.)

The $J$-holomorphic curves in $X_{r}(f)$ give cycles in $\hat{X}$, but there is no reason these should be symplectic. We are trying to build standard surfaces, i.e. we need our cycles to have positive simple tangencies to fibers. This is exactly saying we want sections of $X_{r}(f)$ transverse to the diagonal locus $\Delta$ and intersecting it positively. To achieve this, we will need to construct almost complex structures which behave well with respect to $\Delta$, which has a natural stratification by the combinatorial type of the tuple. Problems arise because we cannot a priori suppose that our sections do not have image entirely contained inside the diagonals.

As a first delicacy, the diagonal strata are not smoothly embedded, but are all the images of smooth maps which are generically bijective. This leads to "smooth models" of the diagonal strata, and for suitable $J$ any holomorphic section will lift to a unique such model. (In particular, if a given section lies inside $\Delta$ because it contains multiple components, then we "throw away" this multiplicity; restoring it later will mean that we construct positive symplectic divisors, in the first instance, and not embedded submanifolds.) As a second delicacy, we haven't controlled the index of sections once we lift them to smooth models of the strata. Finally, although we are restricting to the class of almost complex structures which are compatible with $\Delta$, nonetheless we hope to find holomorphic sections transverse to $\Delta$ itself. The result we need, then, can loosely be summed up as:

Proposition 4.12. There are "enough" almost complex structures compatible with the strata. 
A careful proof is given in [DS], Sections 6-7. The key is that we can obtain deformations of such almost complex structures from vector fields on the fibers of $f$. Since we can find a vector field on $\mathbb{C}$ taking any prescribed values at a fixed set of points, once we restrict to an open subset of the graph of any given holomorphic section which lies in the top stratum of its associated model there are enough deformations to generate the whole tangent space to the space of $J$ which are both fibered and compatible with $\Delta$.

Remark. There are also strata coming from exceptional sections inside $X$, each with fiber $S y m^{2 g-3}$. These also vary topologically trivially, and we repeat the above discussion for them. This lets us get standard surfaces in $\hat{X} \backslash \cup E_{i} \cong X \backslash\left\{b_{j}\right\}$.

The upshot is that the nonvanishing of the Gromov invariant for the class $h \in$ $H_{2}\left(X_{r}(f)\right)$ means we can find a holomorphic section of $X_{r}(f)$ which is transverse and positive to all strata of the diagonal in which it is not contained. Taking into account the strata coming from exceptional sections as well, and with care, one obtains a positive symplectic divisor $D=\sum a_{i} \Sigma_{i}$ in $X$ in the homology class dual to $K_{X}$. We must finally check that we can satisfy the conditions of our earlier smoothing result. By adjunction,

$$
D \cdot \Sigma_{j}+\Sigma_{j}^{2}=2 g\left(\Sigma_{j}\right)-2=\left(1+a_{j}\right) \Sigma_{j}^{2}+\sum_{i \neq j} a_{i} \Sigma_{i} \cdot \Sigma_{j}
$$

For $D \cdot \Sigma_{j}$ to be negative, it must be that $\Sigma_{j}$ is a rational -1 curve; but clearly it suffices to prove the theorem under the assumption that $X$ is minimal. Provided $b_{+}>1+b_{1}(X)$, we therefore obtain an embedded symplectic surface in $X$ representing the class $K_{X}$. (Strictly we have proven this theorem only when $\omega_{X}$ is rational, but one can remove this assumption, cf. [Sm4.)

This result gives "gauge-theory free" proofs of various standard facts on symplectic four-manifolds: for instance, if $b_{+}(X)>1+b_{1}(X)$ and $X$ is minimal, then $c_{1}^{2}(X) \geq 0$. In particular, manifolds such as $K 3 \# K 3 \# K 3$ admit no symplectic structure, illustrating the claim (made in the Introduction) that the symplectic condition cannot be reduced to cohomological or homotopical conditions, in contrast to the case of open manifolds covered by Gromov's h-principle.

To close, we point out that we can also see Taubes' remarkable duality $\operatorname{Gr}(D)=$ $\pm G r(K-D)$ very geometrically in this picture. Let $\iota: \operatorname{Pic}^{2 g-2-r}(\Sigma) \rightarrow \operatorname{Pic}^{r}(\Sigma)$ denote the map $O(D) \mapsto O(K-D)$ and write $\tau_{d}$ for the Abel-Jacobi map $\operatorname{Sym}^{d}(\Sigma) \rightarrow$ $\operatorname{Pic}^{d}(\Sigma)$. If $r$ is small relative to $g$, then generically we expect the map $\tau_{r}$ to be an embedding, and its image to be exactly where the map $\iota \circ \tau_{2 g-2-r}$ has fibers of excess dimension. If we fix a homology class on $X$ and take pencils of higher and higher degree, we can make $r$ (the intersection number with the fiber) as small as we like relative to $g$ (the genus of the fiber) - the latter grows quadratically, not linearly. Combining this with some deep results in the Brill-Noether theory of Riemann surfaces, one can show the following generalisation of Proposition 4.8

Proposition 4.13 ( $\mathrm{Sm} 4)$. For $r$ small enough relative to $g$, there exists $J$ on $X_{2 g-2-r}(f)$ such that $X_{r}(f)$ is embedded in $P_{r}(f) \cong P_{2 g-2-r}(f)$, and such that $X_{2 g-2-r}(f) \rightarrow P_{2 g-2-r}(f)$ is holomorphic and locally trivial over $X_{r}(f)$ and its complement. 
Using this and repeating all the above, we can show there are well-defined integervalued invariants $\mathcal{I}_{f}$ counting sections of the $X_{r}(f)$ 's which satisfy the duality

$$
\mathcal{I}_{f}(D)= \pm \mathcal{I}_{f}\left(K_{X}-D\right) \text {. }
$$

In fact, a smooth fibered $J$ on $X$ defines a canonical fibered $\mathbb{J}$ on $X_{r}(f)$ but this is only $C^{0}$ along the diagonal strata. Suitably interpreted, $J$-curves in $X$ and $\mathbb{J}$ curves in $X_{r}(f)$ are tautologically equivalent. Motivated by this, and a (very rough) sketch showing $\mathcal{I}_{f}=G r$ mod 2 for symplectic manifolds with $K_{X}=\lambda[\omega]$ for nonzero $\lambda$ (more generally whenever there are no embedded square zero symplectic tori), Sm4 conjectured that the $\mathcal{I}$-invariants and Taubes' Gromov invariants coincide. Recent work of Michael Usher Us clarifies and completes this circle of ideas by showing that in full generality $G r=\mathcal{I}_{f}$ (in $\mathbb{Z}$ ) for any pencil $f$ of sufficiently

high degree. In this sense, the above equation shows that Taubes' duality can be understood in terms of Serre duality on the fibers of a Lefschetz fibration.

\section{FukAYA CATEgORIES AND LEFschetZ FibRATions}

Many central questions in symplectic topology revolve around Lagrangian submanifolds, their existence and their intersection properties. From a formal point of view these can be encoded into so-called Fukaya categories, which also play an important role of their own in Kontsevich's homological mirror symmetry conjecture. It turns out that Lefschetz pencils provide some of the most powerful tools available in this context, if one considers vanishing cycles and the "matching paths" between them [Se4]; the most exciting applications to this date are related to Seidel's construction of "directed Fukaya categories" of vanishing cycles [Se1, Se2], and to verifications of the homological mirror conjecture [Se5].

Fukaya categories of symplectic manifolds are intrinsically very hard to compute, because relatively little is known about embedded Lagrangian submanifolds in symplectic manifolds of dimension 4 or more, especially in comparison to the much better understood theory of coherent sheaves over complex varieties, which play the role of their mirror counterparts. The input provided by Lefschetz fibrations is a reduction from the symplectic geometry of the total space to that of the fiber, which in the 4-dimensional case provides a crucial simplification.

5.1. Matching paths and Lagrangian spheres. Let $f: X \rightarrow S^{2}$ be a symplectic Lefschetz fibration (e.g. obtained by blowing up the base points of a Lefschetz pencil or, allowing the fibers to be open, by simply removing them), and let $\gamma \subset S^{2}$ be an embedded arc joining a regular value $p_{0}$ to a critical value $p_{1}$, avoiding all the other critical values of $f$. Using the horizontal distribution given by the symplectic orthogonal to the fibers, we can transport the vanishing cycle at $p_{1}$ along the arc $\gamma$ to obtain a Lagrangian disc $D_{\gamma} \subset X$ fibered above $\gamma$, whose boundary is an embedded Lagrangian sphere $S_{\gamma}$ in the fiber $\Sigma_{0}=f^{-1}\left(p_{0}\right)$. The Lagrangian disc $D_{\gamma}$ is called the Lefschetz thimble over $\gamma$, and its boundary $S_{\gamma}$ is the vanishing cycle already considered in Section 2.

If we now consider an arc $\gamma$ joining two critical values $p_{1}, p_{2}$ of $f$ and passing through $p_{0}$, then the above construction applied to each half of $\gamma$ yields two Lefschetz thimbles $D_{1}$ and $D_{2}$, whose boundaries are Lagrangian spheres $S_{1}, S_{2} \subset \Sigma_{0}$. If $S_{1}$ and $S_{2}$ coincide exactly, then $D_{1} \cup D_{2}$ is an embedded Lagrangian sphere in $X$, fibering above the arc $\gamma$ (see the picture below); more generally, if $S_{1}$ and $S_{2}$ are Hamiltonian isotopic to each other, then perturbing slightly the symplectic 
structure we can reduce to the previous case and obtain again a Lagrangian sphere in $X$. The $\operatorname{arc} \gamma$ is called a matching path in the Lefschetz fibration $f \underline{\text { Se4 }}$.

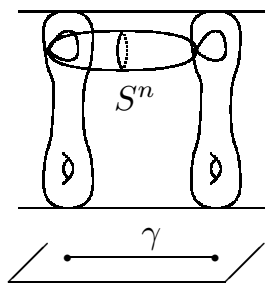

Matching paths are an important source of Lagrangian spheres, and more generally (extending suitably the notion of matching path to embedded arcs passing through several critical values of $f$ ) of embedded Lagrangian submanifolds. Conversely, a folklore theorem asserts that any given embedded Lagrangian sphere in a compact symplectic manifold is isotopic to one that fibers above a matching path in a Donaldson-type symplectic Lefschetz pencil of sufficiently high degree. More precisely, the following result holds (see AMP for a detailed proof):

Theorem 5.1. Let $L$ be a compact Lagrangian submanifold in a compact symplectic manifold $(X, \omega)$ with integral $[\omega]$, and let $h: L \rightarrow[0,1]$ be any Morse function. Then for large enough $k$ there exist Donaldson pencils $f_{k}: X-\{$ base points $\} \rightarrow S^{2}$, embedded arcs $\gamma_{k}:[0,1] \hookrightarrow S^{2}$, and Morse functions $h_{k}: L \rightarrow[0,1]$ isotopic to $h$, such that the restriction of $f_{k}$ to $L$ is equal to $\gamma_{k} \circ h_{k}$.

The intersection theory of Lagrangian spheres that fiber above matching paths is much nicer than that of arbitrary Lagrangian spheres, because if two Lagrangian spheres $S, S^{\prime} \subset X$ fiber above matching paths $\gamma, \gamma^{\prime}$, then all intersections of $S$ with $S^{\prime}$ lie in the fibers above the intersection points of $\gamma$ with $\gamma^{\prime}$. Hence, the Floer homology of $S$ and $S^{\prime}$ can be computed by studying intersection theory for Lagrangian spheres in the fibers of $f$ rather than in $X$. In particular, when $X$ is a four-manifold the vanishing cycles are just closed loops in Riemann surfaces, and the computation of Floer homology essentially reduces to a combinatorial count.

The enumeration of matching paths, if possible, would lead to a complete understanding of isotopy classes of Lagrangian spheres in a given symplectic manifold, with various topological consequences and applications to the definition of new symplectic invariants. However, no finite-time algorithm is currently available for this problem, although some improvements on the "naive" search are possible (e.g. using maps to $\mathbb{C P}^{2}$ and projective duality to identify certain types of pencil automorphisms). Nonetheless, Theorem [5.1 roughly says that all Lagrangians are built out of Lefschetz thimbles. This implies that, at the formal level of (derived) Fukaya categories, it is sometimes possible to identify a "generating" collection of Lagrangian submanifolds (out of which all others can be built by gluing operations, or, in the language of categories, by passing to bounded complexes). A spectacular illustration is provided by Seidel's recent verification of homological mirror symmetry for quartic K3 surfaces Se5.

5.2. Fukaya categories of vanishing cycles. The above considerations, together with ideas of Kontsevich about mirror symmetry for Fano varieties, have led Seidel to the following construction of a Fukaya-type $A_{\infty}$-category associated to a symplectic Lefschetz pencil $f$ on a compact symplectic manifold $(X, \omega)$ Se1. Let $f$ 
be a symplectic Lefschetz pencil determined by two sections $s_{0}, s_{1}$ of a sufficiently positive line bundle $L^{\otimes k}$ as in Theorem 2.9. Assume that $\Sigma_{\infty}=s_{1}^{-1}(0)$ is a smooth fiber of the pencil, and consider the symplectic manifold with boundary $X^{0}$ obtained from $X$ by removing a suitable neighborhood of $\Sigma_{\infty}$. The map $f$ induces a Lefschetz fibration $f^{0}: X^{0} \rightarrow D^{2}$ over a disc, whose fibers are symplectic submanifolds with boundary obtained from the fibers of $f$ by removing a neighborhood of their intersection points with the symplectic hypersurface $\Sigma_{\infty}$ (the base points of the pencil). Choose a reference point $p_{0} \in \partial D^{2}$, and consider the fiber $\Sigma_{0}=\left(f^{0}\right)^{-1}\left(p_{0}\right) \subset X^{0}$.

Let $\gamma_{1}, \ldots, \gamma_{r}$ be a collection of arcs in $D^{2}$ joining the reference point $p_{0}$ to the various critical values of $f^{0}$, intersecting each other only at $p_{0}$, and ordered in the clockwise direction around $p_{0}$. As discussed above, each arc $\gamma_{i}$ gives rise to a Lefschetz thimble $D_{i} \subset X^{0}$, whose boundary is a Lagrangian sphere $L_{i} \subset \Sigma_{0}$. To avoid having to discuss the orientation of moduli spaces, we give the following definition using $\mathbb{Z}_{2}$ (instead of $\mathbb{Z}$ ) as the coefficient ring Se1]:

Definition 5.2 (Seidel). The Fukaya category of vanishing cycles $\mathcal{F}_{v c}\left(f ;\left\{\gamma_{i}\right\}\right)$ is a (directed) $A_{\infty}$-category with $r$ objects $L_{1}, \ldots, L_{r}$ (corresponding to the vanishing cycles, or more accurately to the thimbles); the morphisms between the objects are given by

$$
\operatorname{Hom}\left(L_{i}, L_{j}\right)= \begin{cases}C F^{*}\left(L_{i}, L_{j} ; \mathbb{Z}_{2}\right)=\mathbb{Z}_{2}^{\left|L_{i} \cap L_{j}\right|} & \text { if } i<j \\ \mathbb{Z}_{2} i d & \text { if } i=j \\ 0 & \text { if } i>j\end{cases}
$$

and the differential $\mu^{1}$, composition $\mu^{2}$ and higher order compositions $\mu^{n}$ are given by Lagrangian Floer homology inside $\Sigma_{0}$. More precisely,

$$
\mu^{n}: \operatorname{Hom}\left(L_{i_{0}}, L_{i_{1}}\right) \otimes \cdots \otimes \operatorname{Hom}\left(L_{i_{n-1}}, L_{i_{n}}\right) \rightarrow \operatorname{Hom}\left(L_{i_{0}}, L_{i_{n}}\right)[2-n]
$$

is trivial when the inequality $i_{0}<i_{1}<\cdots<i_{n}$ fails to hold (i.e. it is always zero in this case, except for $\mu^{2}$ where composition with an identity morphism is given by the obvious formula). When $i_{0}<\cdots<i_{n}, \mu^{n}$ is defined by counting pseudoholomorphic maps from the disc to $\Sigma_{0}$, mapping $n+1$ cyclically ordered marked points on the boundary to the given intersection points between vanishing cycles, and the portions of boundary between them to $L_{i_{0}}, \ldots, L_{i_{n}}$.

One of the most attractive features of this definition is that it only involves Floer homology for Lagrangians inside the hypersurface $\Sigma_{0}$; in particular, when $X$ is a symplectic 4-manifold, the definition becomes purely combinatorial, since in the case of a Riemann surface the pseudo-holomorphic discs appearing in the definition of Floer homology and product structures are just immersed polygonal regions with convex corners.

From a technical point of view, a property that greatly facilitates the definition of Floer homology for the vanishing cycles $L_{i}$ is exactness. Namely, the symplectic structure on the manifold $X^{0}$ is exact, i.e. it can be expressed as $\omega=d \theta$ for some 1-form $\theta$ (up to a scaling factor, $\theta$ is the 1-form describing the connection on $L^{\otimes k}$ in the trivialization of $L^{\otimes k}$ over $X-\Sigma_{\infty}$ induced by the section $\left.s_{1} /\left|s_{1}\right|\right)$. With this understood, the submanifolds $L_{i}$ are all exact Lagrangian, i.e. the restriction $\theta_{\mid L_{i}}$ is not only closed $\left(d \theta_{\mid L_{i}}=\omega_{\mid L_{i}}=0\right)$ but also exact, $\theta_{\mid L_{i}}=d \phi_{i}$. Exactness has two particularly nice consequences. First, $\Sigma^{0}$ contains no closed pseudo-holomorphic curves (because the cohomology class of $\omega=d \theta$ vanishes). Secondly, there are no non-trivial pseudo-holomorphic discs in $\Sigma_{0}$ with boundary contained in one of the 
Lagrangian submanifolds $L_{i}$. Indeed, for any such disc $D$, we have $\operatorname{Area}(D)=$ $\int_{D} \omega=\int_{\partial D} \theta=\int_{\partial D} d \phi_{i}=0$. Therefore, bubbling never occurs (neither in the interior nor on the boundary of the domain) in the moduli spaces used to define the Floer homology groups $H F\left(L_{i}, L_{j}\right)$. Moreover, the exactness of $L_{i}$ provides a priori estimates on the area of all pseudo-holomorphic discs contributing to the definition of the products $\mu^{n}(n \geq 1)$; this implies the finiteness of the number of discs to be considered and solves elegantly the convergence problems that normally make it necessary to define Floer homology over Novikov rings.

Example. We now illustrate the above definition by considering the example of a pencil of degree 2 curves in $\mathbb{C P}^{2}$ (see also [e2]). Consider the two sections $s_{0}=x_{0}\left(x_{1}-x_{2}\right)$ and $s_{1}=x_{1}\left(x_{2}-x_{0}\right)$ of the line bundle $O(2)$ over $\mathbb{C P}^{2}$ : their zero sets are singular conics, in fact the unions of two lines each containing two of the four intersection points $(1: 0: 0),(0: 1: 0),(0: 0: 1),(1: 1: 1)$. Moreover, the zero set of the linear combination $s_{0}+s_{1}=x_{2}\left(x_{1}-x_{0}\right)$ is also singular; on the other hand, it is fairly easy to check that all other linear combinations $s_{0}+\alpha s_{1}$ (for $\alpha \in \mathbb{C P}^{1}-\{0,1, \infty\}$ ) define smooth conics. Removing a neighborhood of a smooth fiber of the pencil generated by $s_{0}$ and $s_{1}$, we obtain a Lefschetz fibration over the disc, with fiber a sphere with four punctures. The three singular fibers of the pencil are nodal configurations consisting of two tranversely intersecting spheres, with each component containing two of the four base points; each of the three different manners in which four points can be split into two groups of two is realized at one of the singular fibers. The following diagram represents the three singular conics of the pencil inside $\mathbb{C P}^{2}$ (left), and the corresponding vanishing cycles inside a smooth fiber (right):
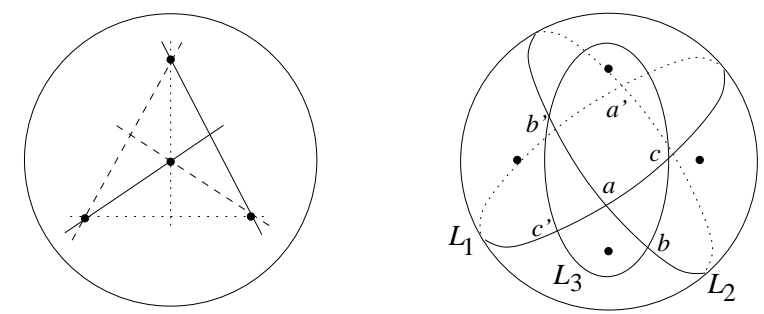

We can describe the monodromy of this Lefschetz pencil by a homomorphism $\psi: \pi_{1}\left(\mathbb{C}-\left\{p_{1}, p_{2}, p_{3}\right\}\right) \rightarrow \mathrm{Map}_{0,4}$ with values in the mapping class group of a genus 0 surface with 4 boundary components. After choosing a suitable ordered basis of the free group $\pi_{1}\left(\mathbb{C}-\left\{p_{1}, p_{2}, p_{3}\right\}\right)$, we can make sure that $\psi$ maps the generators to the Dehn twists $\tau_{1}, \tau_{2}, \tau_{3}$ along the three loops shown on the diagram. On the other hand, because the normal bundles to the exceptional sections of the blown-up Lefschetz fibration have degree -1 , the monodromy at infinity is given by the boundary twist $\prod \delta_{i}$, the product of the four Dehn twists along small loops encircling the four base points in the fiber; on the other hand it is also the product of the monodromies around each of the three singular fibers $\left(\tau_{1}, \tau_{2}, \tau_{3}\right)$. Hence, the monodromy of a pencil of conics in $\mathbb{C P}^{2}$ can be expressed by the relation $\prod \delta_{i}=\tau_{1} \cdot \tau_{2} \cdot \tau_{3}$ in the mapping class group $\operatorname{Map}_{0,4}$ (lantern relation).

Any two of the three vanishing cycles intersect transversely in two points, so $\operatorname{Hom}\left(L_{1}, L_{2}\right)=\mathbb{Z}_{2} a \oplus \mathbb{Z}_{2} a^{\prime}, \operatorname{Hom}\left(L_{2}, L_{3}\right)=\mathbb{Z}_{2} b \oplus \mathbb{Z}_{2} b^{\prime}$, and $\operatorname{Hom}\left(L_{1}, L_{3}\right)=$ $\mathbb{Z}_{2} c \oplus \mathbb{Z}_{2} c^{\prime}$ are all two-dimensional. There are no immersed 2-sided polygons in 
the punctured sphere $\Sigma_{0}$ with boundary in $L_{i} \cup L_{j}$ for any pair $(i, j)$, since each of the four regions delimited by $L_{i}$ and $L_{j}$ contains one of the punctures, so $\mu^{1} \equiv 0$. However, there are four triangles with boundary in $L_{1} \cup L_{2} \cup L_{3}$ (with vertices $a b c$, $a b^{\prime} c^{\prime}, a^{\prime} b^{\prime} c, a^{\prime} b c^{\prime}$ respectively), and in each case the cyclic ordering of the boundary is compatible with the ordering of the vanishing cycles. Therefore, the composition of morphisms is given by the formulas $\mu^{2}(a, b)=\mu^{2}\left(a^{\prime}, b^{\prime}\right)=c, \mu^{2}\left(a, b^{\prime}\right)=\mu^{2}\left(a^{\prime}, b\right)=$ $c^{\prime}$. Finally, the higher compositions $\mu^{n}, n \geq 3$ are all trivial in this category, because the ordering condition $i_{0}<\cdots<i_{n}$ never holds Se2].

The objects $L_{i}$ of the category $\mathcal{F}_{v c}\left(f ;\left\{\gamma_{i}\right\}\right)$ actually correspond not only to Lagrangian spheres in $\Sigma_{0}$ (the vanishing cycles), but also to Lagrangian discs in $X^{0}$ (the Lefschetz thimbles $D_{i}$ ); and the Floer intersection theory in $\Sigma_{0}$ giving rise to $\operatorname{Hom}\left(L_{i}, L_{j}\right)$ and to the product structures can also be thought of in terms of intersection theory for the thimbles $D_{i}$ in $X^{0}$ (this is actually the reason of the asymmetry between the cases $i<j$ and $i>j$ in Definition [5.2). In any case, the properties of these objects depend very much on the choice of the ordered collection of arcs $\left\{\gamma_{i}\right\}$. Therefore, $\mathcal{F}_{v c}\left(f ;\left\{\gamma_{i}\right\}\right)$ has little geometric meaning in itself, and should instead be viewed as a collection of generators of a much larger category which includes not only the Lefschetz thimbles, but also more general Lagrangian submanifolds of $X^{0}$. More precisely, the category naturally associated to the Lefschetz pencil $f$ is not the finite directed $A_{\infty}$-category defined above, but rather the (split-closed) derived category $D \mathcal{F}_{v c}(f)$ obtained from $\mathcal{F}_{v c}\left(f ;\left\{\gamma_{i}\right\}\right)$ by considering (twisted) complexes of formal direct sums of objects (also including idempotent splittings and formal inverses of quasi-isomorphisms). Replacing the ordered collection $\left\{\gamma_{i}\right\}$ by another one $\left\{\gamma_{i}^{\prime}\right\}$ leads to a different "presentation" of the same derived category. Indeed, we have the following result [Se1]:

Theorem 5.3 (Seidel). Given any two ordered collections $\left\{\gamma_{i}\right\}$ and $\left\{\gamma_{i}^{\prime}\right\}$, the categories $\mathcal{F}_{v c}\left(f ;\left\{\gamma_{i}\right\}\right)$ and $\mathcal{F}_{v c}\left(f ;\left\{\gamma_{i}^{\prime}\right\}\right)$ differ by a sequence of mutations (operations that modify the ordering of the objects while twisting some of them along others). Hence, the derived category $D \mathcal{F}_{v c}(f)$ does not depend on the choice of $\left\{\gamma_{i}\right\}$.

Roughly speaking, complexes in the derived category correspond to Lagrangian cycles obtained by gluing the corresponding Lefschetz thimbles. For example, assume that the vanishing cycles $L_{i}$ and $L_{j}(i<j)$ are Hamiltonian isotopic to each other, so that (a smoothing of) $\gamma_{i} \cup \gamma_{j}$ is a matching path. Then $\operatorname{Hom}\left(L_{i}, L_{j}\right)$ has rank two, with one generator in degree 0 and one in degree $n-1=\operatorname{dim} L_{i}$; let $a$ be the degree 0 generator. Then the complex $C=\left\{0 \rightarrow L_{i} \stackrel{a}{\longrightarrow} L_{j} \rightarrow 0\right\}$, viewed as an object of the derived category, represents the Lagrangian sphere associated to the matching path $\gamma_{i} \cup \gamma_{j}$; for example it is easy to check that $\operatorname{Hom}_{D \mathcal{F}_{v c}}(C, C) \simeq H^{*}\left(S^{n}, \mathbb{Z}_{2}\right)$.

Building Fukaya-type categories out of vanishing cycles may seem arbitrary, but has a solid geometric underpinning, as suggested by the discussion at the end of §5.1. In fairly general circumstances, every closed Lagrangian submanifold (with well-defined and non-zero Floer homology with itself) of a Kähler manifold $X$ must intersect one of the vanishing cycles of any Lefschetz pencil containing $X$ as a smooth member. For the theory of Biran and Cieliebak [BC] shows that otherwise such a submanifold could be displaced off itself by a Hamiltonian isotopy (first by moving the Lagrangian into an open domain in $X$ admitting a plurisubharmonic function with no top index critical points), a contradiction to the non-triviality 
of Floer homology. Seidel has pushed this even further: for certain K3 surfaces arising as anticanonical divisors in Fano 3 -folds, every Lagrangian submanifold $L$ must have non-trivial Floer cohomology with one of the vanishing cycles of the pencil. Otherwise, by repeatedly applying the exact sequence in Floer cohomology, one sees that $H F(L, L)$ is graded isomorphic to a shifted version of itself, which is absurd. In this sense, the vanishing cycles really do "generate" the Fukaya category; if $H F(L, V)=0$ for all vanishing cycles $V$, that is if $L$ has no morphisms to any vanishing cycle, then $H F(L, L)=0$ so $L$ has no identity morphism, and must represent the zero object of the category.

5.3. Applications to mirror symmetry. The construction described above has various applications to homological mirror symmetry. In the context of CalabiYau manifolds, these have to do with a conjecture of Seidel about the relationship between the derived Fukaya category $D \mathcal{F}_{v c}(f)$ of the Lefschetz pencil $f$ and the derived Fukaya category $D \mathcal{F}(X)$ of the closed symplectic manifold $X$ Se3]. As seen above, when passing to the derived category $D \mathcal{F}_{v c}(f)$, we hugely increase the number of objects, by considering not only the thimbles $D_{i}$ but also arbitrary complexes obtained from them; this means that the objects of $D \mathcal{F}_{v c}(f)$ include all sorts of (not necessarily closed) Lagrangian submanifolds in $X^{0}$, with boundary in $\Sigma_{0}$. Since Fukaya categories are only concerned with closed Lagrangian submanifolds, it is necessary to consider a subcategory of $D \mathcal{F}_{v c}(f)$ whose objects correspond to the closed Lagrangian submanifolds in $X^{0}$ (i.e., combinations of $D_{i}$ for which the boundaries cancel); it is expected that this can be done in purely categorical terms by considering those objects of $D \mathcal{F}_{v c}(f)$ on which the Serre functor acts simply by a shift. The resulting subcategory should be closely related to the derived Fukaya category of the open manifold $X^{0}$. This leaves us with the problem of relating $\mathcal{F}\left(X^{0}\right)$ with $\mathcal{F}(X)$. These two categories have the same objects and morphisms (Lagrangians in $X$ can be made disjoint from $\Sigma_{\infty}$ ), but the differentials and product structures differ. More precisely, the definition of $\mu^{n}$ in $\mathcal{F}\left(X^{0}\right)$ only involves counting pseudo-holomorphic discs contained in $X^{0}$, i.e. disjoint from the hypersurface $\Sigma_{\infty}$. In order to account for the missing contributions, one should introduce a formal parameter $q$ and count the pseudo-holomorphic discs with boundary in $\bigcup L_{i}$ that intersect $\Sigma_{\infty}$ in $m$ points (with multiplicities) with a coefficient $q^{m}$. The introduction of this parameter $q$ leads to a deformation of $A_{\infty}$-structures, i.e. an $A_{\infty}$-category in which the differentials and products $\mu^{n}$ are defined over a ring of formal power series in the variable $q$; the limit $q=0$ corresponds to the (derived) Fukaya category $D \mathcal{F}\left(X^{0}\right)$, while non-zero values of $q$ are expected to yield $D \mathcal{F}(X)$.

These considerations provide a strategy to calculate Fukaya categories (at least for some examples) by induction on dimension $\underline{\mathrm{Se} 3}$; an important recent development in this direction is Seidel's proof of homological mirror symmetry for quartic K3 surfaces [Se5].

Returning to more elementary considerations, another context in which the construction of Definition 5.2 is relevant is that of mirror symmetry for Fano manifolds $\left(c_{1}(T M)>0\right)$. Rather than manifolds, mirrors of Fano manifolds are Landau-Ginzburg models, i.e. pairs $(Y, w)$, where $Y$ is a non-compact manifold and $w: Y \rightarrow \mathbb{C}$, the "superpotential", is a holomorphic function. The complex (resp. symplectic) geometry of a Fano manifold $M$ is then expected to correspond to the symplectic (resp. complex) geometry of the critical points of the superpotential $w$ 
on its mirror. In particular, the homological mirror conjecture of Kontsevich now asserts that the categories $D^{b} \operatorname{Coh}(M)$ and $D \mathcal{F}_{v c}(w)$ should be equivalent. Following the ideas of various people (Kontsevich, Hori, Vafa, Seidel, etc.), the conjecture can be verified on many examples, at least in those cases where the critical points of $w$ are isolated and non-degenerate.

For example, let $a, b, c$ be three mutually prime positive integers, and consider the weighted projective plane $M=\mathbb{C P}^{2}(a, b, c)=\left(\mathbb{C}^{3}-\{0\}\right) / \mathbb{C}^{*}$, where $\mathbb{C}^{*}$ acts by $t \cdot(x, y, z)=\left(t^{a} x, t^{b} y, t^{c} z\right)$. In general $M$ is a Fano orbifold (when $a=b=c=1$ it is the usual projective plane). Consider the mirror Landau-Ginzburg model $(Y, w)$, where $Y=\left\{x^{a} y^{b} z^{c}=1\right\}$ is a hypersurface in $\left(\mathbb{C}^{*}\right)^{3}$, equipped with an exact Kähler form, and $w=x+y+z$. The superpotential $w$ has $a+b+c$ isolated non-degenerate critical points, and hence determines an affine Lefschetz fibration to which we can apply the construction of Definition 5.2 To be more precise, in this context one should actually define the Fukaya category of vanishing cycles over a coefficient ring $R$ larger than $\mathbb{Z}_{2}$ or $\mathbb{Z}$, for example $R=\mathbb{C}$, counting each pseudo-holomorphic disc $u: D^{2} \rightarrow \Sigma_{0}$ with a weight $\pm \exp \left(-\int_{D^{2}} u^{*} \omega\right)$, or a Novikov ring. Then we have the following result:

Theorem 5.4 (AKO). The categories $D^{b} \operatorname{Coh}(M)$ and $D \mathcal{F}_{v c}(w)$ are equivalent.

The proof relies on the identification of suitable collections of generators for both categories, and an explicit verification that the morphisms on both sides obey the same composition rules. Moreover, it can also be shown that non-exact deformations of the Kähler structure on $Y$ correspond to non-commutative deformations of $M$, with an explicit relationship between the deformation parameter on $M$ and the complexified Kähler class $[\omega+i B] \in H^{2}(Y, \mathbb{C})$ AKO.

The homological mirror conjecture can be similarly verified for various other examples, and one may reasonably expect that, in the near future, vanishing cycles will play an important role in our understanding of mirror symmetry, not only for Fano and Calabi-Yau varieties, but maybe also for varieties of general type.

\section{REFERENCES}

[ABKP] J. Amorós, F. Bogomolov, L. Katzarkov, T. Pantev, Symplectic Lefschetz fibrations with arbitrary fundamental groups, J. Differential Geom. 54 (2000), 489-545.

[AGTV] M. Amram, D. Goldberg, M. Teicher, U. Vishne, The fundamental group of the Galois cover of the surface $\mathbb{C P}^{1} \times T$, Alg. Geom. Topol. 2 (2002), 403-432.

[Au1] D. Auroux, Asymptotically holomorphic families of symplectic submanifolds, Geom. Funct. Anal. 7 (1997), 971-995.

[Au2] D. Auroux, Symplectic 4-manifolds as branched coverings of $\mathbb{C P}^{2}$, Invent. Math. 139 (2000), 551-602.

[Au3] D. Auroux, Symplectic maps to projective spaces and symplectic invariants, Turkish J. Math. 25 (2001), 1-42 (math.GT/0007130).

[Au4] D. Auroux, Estimated transversality in symplectic geometry and projective maps, "Symplectic Geometry and Mirror Symmetry", Proc. 4th KIAS International Conference, Seoul (2000), World Sci., Singapore, 2001, pp. 1-30 (math.SG/0010052).

[Au5] D. Auroux, A remark about Donaldson's construction of symplectic submanifolds, J. Symplectic Geom. 1 (2002), 647-658 (math.DG/0204286).

[Au6] D. Auroux, Fiber sums of genus 2 Lefschetz fibrations, Turkish J. Math. 27 (2003), 1-10 (math.GT/0204285).

[ADK] D. Auroux, S. K. Donaldson, L. Katzarkov, Luttinger surgery along Lagrangian tori and non-isotopy for singular symplectic plane curves, Math. Ann. 326 (2003), 185-203. 
[ADKY] D. Auroux, S. K. Donaldson, L. Katzarkov, M. Yotov, Fundamental groups of complements of plane curves and symplectic invariants, preprint, to appear in Topology (math.GT/0203183).

[AK] D. Auroux, L. Katzarkov, Branched coverings of $\mathbb{C P}^{2}$ and invariants of symplectic 4manifolds, Invent. Math. 142 (2000), 631-673.

[AK2] D. Auroux, L. Katzarkov, The degree doubling formula for braid monodromies and Lefschetz pencils, preprint.

[AKO] D. Auroux, L. Katzarkov, D. Orlov, Homological mirror symmetry for weighted projective planes, in preparation.

[AMP] D. Auroux, V. Muñoz, F. Presas, Lagrangian submanifolds and Lefschetz pencils, in preparation.

[Bi] P. Biran, A stability property of symplectic packing in dimension 4, Invent. Math. 136 (1999), 123-155.

[BC] P. Biran, K. Cieliebak, Symplectic topology on subcritical manifolds, Comment. Math. Helv. 76 (2001), 712-753.

[Bir] J. Birman, Braids, Links and Mapping class groups, Annals of Math. Studies 82, Princeton Univ. Press, Princeton, 1974.

[CMT] C. Ciliberto, R. Miranda, M. Teicher, Pillow degenerations of K3 surfaces, Applications of Algebraic Geometry to Coding Theory, Physics, and Computation, NATO Science Series II, vol. 36, 2001, pp. 53-63.

[Do1] S. K. Donaldson, Symplectic submanifolds and almost-complex geometry, J. Differential Geom. 44 (1996), 666-705.

[Do2] S.K. Donaldson, Lefschetz fibrations in symplectic geometry, Documenta Math., Extra Volume ICM 1998, II, 309-314.

[Do3] S.K. Donaldson, Lefschetz pencils on symplectic manifolds, J. Differential Geom. 53 (1999), 205-236.

[DS] S. Donaldson, I. Smith, Lefschetz pencils and the canonical class for symplectic 4manifolds, Topology 42 (2003), 743-785.

$\left[\mathrm{FO}^{3}\right] \quad$ K. Fukaya, Y.-G. Oh, H. Ohta, K. Ono, Lagrangian intersection Floer theory: Anomaly and obstruction, preprint.

[FS] R. Fintushel, R. Stern, Symplectic surfaces in a fixed homology class, J. Differential Geom. 52 (1999), 203-222.

[Gi] E. Giroux, Géométrie de contact: de la dimension trois vers les dimensions supérieures, Proc. International Congress of Mathematicians, Vol. II (Beijing, 2002), Higher Ed. Press, Beijing, 2002, pp. 405-414.

[Go1] R. E. Gompf, A new construction of symplectic manifolds, Ann. Math. 142 (1995), 527595.

[Go2] R.E. Gompf, A topological characterization of symplectic manifolds, preprint (math.SG/ 0210103).

[GS] R.E. Gompf, A.I. Stipsicz, 4-manifolds and Kirby calculus, Graduate Studies in Math. 20, Amer. Math. Soc., Providence, 1999.

[Gr] M. Gromov, Pseudo-holomorphic curves in symplectic manifolds, Invent. Math. 82 (1985), 307-347.

[IMP] A. Ibort, D. Martinez-Torres, F. Presas, On the construction of contact submanifolds with prescribed topology, J. Differential Geom. 56 (2000), 235-283.

[KK] V. Kharlamov, V. Kulikov, On braid monodromy factorizations, Izvestia Math. 67 (2003), 79-118 (math.AG/0302113).

[Ku] V. Kulikov, On a Chisini conjecture, Izvestia Math. 63 (1999), 1139-1170.

[McS] D. McDuff and D. Salamon, J-holomorphic curves and quantum cohomology, Univ. Lecture Series No. 6, Amer. Math. Soc., Providence, 1994.

[Mo1] B. Moishezon, Complex surfaces and connected sums of complex projective planes, Lecture Notes in Math. 603, Springer, Heidelberg, 1977.

[Mo2] B. Moishezon, Stable branch curves and braid monodromies, Algebraic Geometry (Chicago, 1980), Lecture Notes in Math. 862, Springer, Heidelberg, 1981, pp. 107-192.

[Mo3] B. Moishezon, On cuspidal branch curves, J. Algebraic Geom. 2 (1993), 309-384.

[Mo4] B. Moishezon, The arithmetic of braids and a statement of Chisini, Geometric Topology (Haifa, 1992), Contemp. Math. 164, Amer. Math. Soc., Providence, 1994, pp. 151-175.

[Mo5] B. Moishezon, Topology of generic polynomial maps in complex dimension two, preprint. 
[MRT] B. Moishezon, A. Robb, M. Teicher, On Galois covers of Hirzebruch surfaces, Math. Ann. 305 (1996), 493-539.

[Na] H. Nakajima, Lectures on Hilbert schemes of points on surfaces, Univ. Lecture Series No. 18, Amer. Math. Soc., Providence, 1999.

[Oz] B. Ozbagci, Signatures of Lefschetz fibrations, Pacific J. Math. 202 (2002), 99-118.

[OS] B. Ozbagci, A. Stipsicz, Noncomplex smooth 4-manifolds with genus 2 Lefschetz fibrations, Proc. Amer. Math. Soc. 128 (2000), 3125-3128.

[Ro] A. Robb, On branch curves of algebraic surfaces, Singularities and Complex Geometry (Beijing, 1994), Amer. Math. Soc./Int. Press Stud. Adv. Math. 5, Amer. Math. Soc., Providence, 1997, pp. 193-221.

[Se1] P. Seidel, Vanishing cycles and mutation, Proc. 3rd European Congress of Mathematics (Barcelona, 2000), Vol. II, Progr. Math. 202, Birkhäuser, Basel, 2001, pp. 65-85 (math.SG/0007115).

[Se2] P. Seidel, More about vanishing cycles and mutation, "Symplectic Geometry and Mirror Symmetry", Proc. 4th KIAS International Conference, Seoul (2000), World Sci., Singapore, 2001, pp. 429-465 (math.SG/0010032).

[Se3] P. Seidel, Fukaya categories and deformations, Proc. International Congress of Mathematicians, Vol. II (Beijing, 2002), Higher Ed. Press, Beijing, 2002, pp. 351-360.

[Se4] P. Seidel, Fukaya categories and Picard-Lefschetz theory, in preparation.

[Se5] P. Seidel, Homological mirror symmetry for the quartic surface, preprint (math.SG/ 0310414).

[Sh] V. Shevchishin, On the local version of the Severi problem, preprint (math.AG/0207048).

[ST] B. Siebert, G. Tian, On the holomorphicity of genus two Lefschetz fibrations, preprint, to appear in Ann. Math (math.SG/0305343).

[Sm1] I. Smith, Lefschetz fibrations and the Hodge bundle, Geom. Topol. 3 (1999), 211-233.

[Sm2] I. Smith, Lefschetz pencils and divisors in moduli space, Geom. Topol. 5 (2001), 579-608.

[Sm3] I. Smith, Geometric monodromy and the hyperbolic disc, Quarterly J. Math. 52 (2001), 217-228 (math.SG/0011223).

[Sm4] I. Smith, Serre-Taubes duality for pseudoholomorphic curves, Topology 42 (2003), 931979 .

[Ta1] C. H. Taubes, The Seiberg-Witten and the Gromov invariants, Math. Res. Lett. 2 (1995), 221-238.

[Ta2] C.H. Taubes, The geometry of the Seiberg-Witten invariants, Surveys in Differential Geometry, Vol. III (Cambridge, 1996), Int. Press, Boston, 1998, pp. 299-339.

[Te1] M. Teicher, Braid groups, algebraic surfaces and fundamental groups of complements of branch curves, Algebraic Geometry (Santa Cruz, 1995), Proc. Sympos. Pure Math., 62 (part 1), Amer. Math. Soc., Providence, 1997, pp. 127-150.

[Te2] M. Teicher, New invariants for surfaces, Tel Aviv Topology Conference: Rothenberg Festschrift (1998), Contemp. Math. 231, Amer. Math. Soc., Providence, 1999, pp. 271281 (math.AG/9902152).

[Te3] M. Teicher, The fundamental group of a $\mathbb{C P}^{2}$ complement of a branch curve as an extension of a solvable group by a symmetric group, Math. Ann. 314 (1999) 19-38.

[Th] W. Thurston, Some simple examples of symplectic manifolds, Proc. Amer. Math. Soc. 55 (1976), 467-468.

[Us] M. Usher, The Gromov invariant and the Donaldson-Smith standard surface count, preprint (math.SG/0310450).

Department of Mathematics, M.I.T., Cambridge MA 02139, USA

E-mail address: auroux@math.mit.edu

Centre for Mathematical Sciences, University of Cambridge, Wilberforce Road, CAmbridge CB3 0WB, U.K.

E-mail address: i.smith@dpmms.cam.ac.uk 